\title{
QUANTUM HALL EFFECT ON THE HYPERBOLIC PLANE
}

\author{
A. L. CAREY, K. C. HANNABUSS, V. MATHAi, AND P. MCCANN
}

\begin{abstract}
In this paper, we study both the continuous model and the discrete model of the Quantum Hall Effect (QHE) on the hyperbolic plane. The Hall conductivity is identified as a geometric invariant associated to an imprimitivity algebra of observables. We define a twisted analogue of the Kasparov map, which enables us to use the pairing between $K$-theory and cyclic cohomology theory, to identify this geometric invariant with a topological index, thereby proving the integrality of the Hall conductivity in this case.
\end{abstract}

\section{INTRODUCTION}

The usual model of the integer quantum Hall effect involves electrons moving in a two dimensional conductor under the influence of a magnetic field. The field is applied in a direction orthogonal to the conductor. The Hamiltonian used is that for a single electron moving under the influence of this magnetic field with the addition of a potential term to represent the field due to the lattice of ions making up the conductor. For simplicity this lattice is often assumed to be periodic in the two axis directions in the plane. The effect of impurities can be modelled by departing from a perfectly periodic potential. The definitive treatment from a mathematical point of view is due to Bellisard [Bel+E+S] and Xia Xia]. In this approach no assumption is made about the rationality of the imposed magnetic flux while the integrality of the Hall conductance follows by showing that it is given by the index of a Fredholm operator. Xia also exhibits the conductance as a topological index. These demonstrations use in an essential way Connes' non-commutative differential geometry.

In this paper we are interested in what can be said when one replaces the usual two dimensional conducting material with its Euclidean geometry by a two dimensional sample with hyperbolic geometry. Physically one should think of hyperbolic space and hence the sample as an embedded hyperboloid in Euclidean 3-space. The crystal lattice of the conductor is now modelled by the orbit of a freely acting discrete group. For reasons of convenience we take this to be the fundamental group of a Riemann surface (though aspects of our analysis work more generally). The magnetic field remains orthogonal to the two dimensional conductor and the electric potential we take to be periodic under

Date: FEBRUARY 1997.

1991 Mathematics Subject Classification. Primary: 58G11, 58G18 and 58G25.

Key words and phrases. Quantum Hall Effect, Hyperbolic Space, Riemann surfaces, imprimitivity algebras, $C^{*}$-algebras, $K$-theory, cyclic cohomology, Harper operator. 
the action of this discrete group (we do not attempt to model impurities). When the magnetic flux is rational, spectral properties of Hamiltonians for a single electron have been studied, particularly in the cases where the Hamiltonian may be defined on a finite cover of the Riemann surface [engo+Li]. In this paper however we are interested in approaching the problem using non-commutative geometry, which allows the flux to be any real number, and the algebras of interest are not always associated with vector bundles over the Riemann surface.

We begin by reviewing the construction of the Hamiltonian. First we take as our principal model of hyperbolic space, the hyperbolic plane. This is the upper half-plane $\mathbb{H}$ in $\mathbb{C}$ equipped with its usual Poincaré metric $\left(d x^{2}+d y^{2}\right) / y^{2}$, and symplectic area form $\omega_{\mathbb{H}}=d x \wedge d y / y^{2}$. The group $\mathbf{S L}(2, \mathbb{R})$ acts transitively on $\mathbb{H}$ by Möbius transformations

$$
x+i y=\zeta \mapsto g \zeta=\frac{a \zeta+b}{c \zeta+d}, \quad \text { for } g=\left(\begin{array}{cc}
a & b \\
c & d
\end{array}\right) .
$$

Any Riemann surface of genus $g$ greater than 1 can be realised as the quotient of $\mathbb{H}$ by the action of its fundamental group realised as a $\operatorname{subgroup} \Gamma$ of $\mathbf{S L}(2, \mathbb{Z}) \subset \mathbf{S L}(2, \mathbb{R})$.

Let us now pick a 1-form $\eta$ such that $d \eta=\theta \omega_{\mathbb{H}}$, for some fixed $\theta \in(0,1]$. As in geometric quantisation we may regard $\eta$ as defining a connection $\nabla=d-i \eta$ on a line bundle $\mathcal{L}$ over $\mathbb{H}$, whose curvature is $\theta \omega_{\mathbb{H}}$. Physically we can think of $\eta$ as the electromagnetic vector potential for a uniform magnetic field of strength $\theta$ normal to $\mathbb{H}$. Using the Riemannian metric the Hamiltonian of an electron in this field is given in suitable units by

$$
H=H_{\eta}=-\frac{1}{2} \nabla^{*} \nabla=-\frac{1}{2}(d-i \eta)^{*}(d-i \eta) .
$$

Comtet [Comtet] has shown that $H$ differs from a multiple of the Casimir element for $\mathbf{S L}(2, \mathbb{R}), \frac{1}{8} \mathbf{J} . \mathbf{J}$, by a constant, where $J_{1}, J_{2}$ and $J_{3}$ denote a certain representation of generators of the Lie algebra $\operatorname{sl}(2, \mathbb{R})$, satisfying

$$
\left[J_{1}, J_{2}\right]=-i J_{3}, \quad\left[J_{2}, J_{3}\right]=i J_{1}, \quad\left[J_{3}, J_{1}\right]=i J_{2},
$$

so that $\mathbf{J} . \mathbf{J}=J_{1}^{2}+J_{2}^{2}-J_{3}^{2}$ is the quadratic Casimir element. This shows very clearly the underlying $\mathbf{S L}(2, \mathbb{R})$-invariance of the theory. In a real material this Hamiltonian would be modified by the addition of a potential $V$. By taking $V$ to be invariant under $\Gamma$ this perturbation is given a crystalline type structure analogous to the use of periodic potentials invariant under $\mathbb{Z}^{2}$ in the Euclidean plane $\mathbb{R}^{2}$. Comtet has computed the spectrum of the unperturbed Hamiltonian $H_{\eta}$ for $\eta=-\theta d x / y$ to be the union of finitely many eigenvalues $\left\{(2 k+1) \theta-k(k+1): k=0,1,2 \ldots<\theta-\frac{1}{2}\right\}$, and the continuous spectrum $\left[\frac{1}{4}+\right.$ $\left.\theta^{2}, \infty\right)$ for those values of $\theta$ for which the de Rham cohomology class of $\theta \omega_{\mathbb{H}}$ is integral. Its zeta function and the kernel of its resolvent are also known in this case, Comtet, Comtet $+\mathrm{H}$. Any $\eta$ is cohomologous to $-\theta d x / y$ (since they both have $\omega_{\mathbb{H}}$ as differential) and forms differing by an exact form $d \phi$ give equivalent models: in fact, multiplying the wave functions by $\exp (i \phi)$ shows that the models for $\eta$ and $-\theta d x / y$ are unitarily equivalent. This equivalence also intertwines the $\Gamma$-actions so that the spectral densities for the two models also coincide. However, it is the perturbed Hamiltonian $H_{\eta, V}=H_{\eta}+V$ which is the key to the quantum Hall effect on the hyperbolic plane, and the spectrum of this is unknown for general $\Gamma$-invariant $V$. As we noted above the Hall effect on Riemann 
surfaces has also been considered [engo+Li], $\mathrm{Av}+\mathrm{K}+\mathrm{P}+\mathrm{S}]$ but this is different from the problem we consider here.

These considerations suggest that one could mimic the non-commutative geometry approach of Bellissard-Connes to the integer quantum Hall effect on Euclidean space [Bel], [Nak+Be]], Bel+E+S], Co2], Xia in a hyperbolic setting. This interprets the Hall conductivity as a non-commutative Chern character, whose integrality follows from K-theory. Physically such situations have been considered without the perturbing potential (or $\Gamma$ is trivial) in the context of exploring edge effects for the quantum Hall effect and the behaviour of electrons in quantum dots. Much of the mathematical machinery needed for this has already been discussed in a geometrical context [C]], Co2], Comtet], [Comtet+H] and will be exploited here. We also discuss the discrete version of the theory [Co2], [Sun] motivated in part by some results of [MC] in the Euclidean setting. These hyperbolic Hall effect models occupy the first seven sections. Specifically we show that there is a principal groupoid $C^{*}$-algebra with cocycle for the diagonal $\Gamma$ action on $\mathbb{H} \times \mathbb{H}$ which contains the resolvent of the various Hamiltonians we consider. In order to construct a Fredholm module for this algebra we found it useful to take a more abstract group theoretic approach. We show that our groupoid algebra is isomorphic to a quotient of the $\Gamma$ invariant part of the imprimitivity algebra for inducing from the maximal compact $\operatorname{subgroup~of~} \mathbf{S L}(2, \mathbb{R})$ to $C^{*}(\mathbf{S L}(2, \mathbb{R}), \sigma)$ (the multiplier, or group 2-cocycle, $\sigma$ extends to all of $\left.\mathbf{S L}(2, \mathbb{R})\right)$. This imprimitivity algebra has a regular representation, induced by a canonical trace, the Hilbert space of which provides a Fredholm module which is 2 -summable for a dense subalgebra of the imprimitivity algebra. We show that this dense subalgebra contains the spectral projections corresponding to gaps in the spectrum of our Hamiltonians. Similar results hold in the discrete model as well. The connection between the continuous and discrete models arises from the Morita equivalence of our quotient of the $\Gamma$ invariant imprimitivity algebra with $C^{*}(\Gamma, \sigma)$.

The main results of our paper follow by extending the approach of Xia to cover the hyperbolic case. In fact in Section 8 we prove some general theorems about the $K$-groups of $C^{*}$-algebras which generalise those arising from the hyperbolic Hall effect. The relevance of $K$-theory can be understood in the case of the integer Hall effect on Euclidean space partly as a result of the calculation [Elliott], [Be]], [Co]:

$$
K_{*}\left(C^{*}\left(\mathbb{Z}^{n}, \sigma\right)\right) \cong K_{*}\left(C^{*}\left(\mathbb{Z}^{n}\right)\right) \cong K^{*}\left(\mathbb{T}^{n}\right)
$$

for any multiplier (i.e. group 2-cocycle) $\sigma$ on $\mathbb{Z}^{n}$. This result has lead to the twisted group $C^{*}$-algebras $C^{*}\left(\mathbb{Z}^{n}, \sigma\right)$ being called noncommutative tori. This calculation was generalized by Packer and Raeburn [PR] [PR2], who computed the $K$-groups of the twisted group $C^{*}$ algebras of uniform lattices in solvable groups. More precisely, they proved that if $\Gamma$ is a uniform lattice in a solvable Lie group $G$, then

$$
K_{*}\left(C^{*}(\Gamma, \sigma)\right) \cong K^{*+\operatorname{dim} G}\left(\Gamma \backslash G, \delta\left(B_{\sigma}\right)\right)
$$

where $\sigma$ is any multiplier on $\Gamma, K^{*}\left(\Gamma \backslash G, \delta\left(B_{\sigma}\right)\right)$ denotes the twisted $K$-theory of a continuous trace $C^{*}$-algebra $B_{\sigma}$ with spectrum $\Gamma \backslash G$, while $\delta\left(B_{\sigma}\right) \in H^{3}(\Gamma \backslash G, \mathbb{Z})$ denotes the Dixmier-Douady invariant of $B_{\sigma}$. (Note that the twisted $K$-theory was studied in [Ros]). 
Packer and Raeburn proved a stabilization theorem and used the Thom isomorphism theorem for the $K$-theory of $C^{*}$ algebras, due to Connes [Co2], to prove their results.

In Section 8 we extend the main theorem of [PR], [PR2] to the case when $\Gamma$ is a lattice in a $K$-amenable Lie group $G$. More precisely, we prove that for such $G$ and $\Gamma$,

$$
K_{*}\left(C^{*}(\Gamma, \sigma)\right) \cong K_{*}\left(C_{r}^{*}(\Gamma, \sigma)\right)
$$

and

$$
K_{*}\left(C^{*}(\Gamma, \sigma)\right) \cong K^{*+\operatorname{dim}(G / K)}\left(\Gamma \backslash G / K, \delta\left(B_{\sigma}\right)\right),
$$

where $K$ is a maximal compact subgroup of $G, \sigma$ is any multiplier on $\Gamma, K^{*}\left(\Gamma \backslash G / K, \delta\left(B_{\sigma}\right)\right)$ is the twisted $K$-theory of a continuous trace $C^{*}$-algebra $B_{\sigma}$ with spectrum $\Gamma \backslash G / K$, and $\delta\left(B_{\sigma}\right) \in H^{3}(\Gamma \backslash G / K, \mathbb{Z})$ is the Dixmier-Douady invariant of $B_{\sigma}$.

Our method uses the $K$-amenability results of Kasparov [Kas1] and the Packer-Raeburn stabilization theorem $[\overline{\mathrm{PR}}]$. In the case when $\Gamma=\Gamma_{g}$ is the fundamental group of a Riemann surface $\Sigma_{g}$ of genus $g>0$, we deduce that the Dixmier-Douady class $\delta\left(B_{\sigma}\right)$ ) is trivial. Using this we demonstrate that for any multiplier $\sigma$ on $\Gamma_{g}$

$$
K_{0}\left(C^{*}\left(\Gamma_{g}, \sigma\right)\right) \cong K^{0}\left(\Sigma_{g}\right) \cong \mathbb{Z}^{2}
$$

and that

$$
K_{1}\left(C^{*}\left(\Gamma_{g}, \sigma\right)\right) \cong K^{1}\left(\Sigma_{g}\right) \cong \mathbb{Z}^{2 g}
$$

We end the discussion with an interesting conjecture for compact 3-dimensional manifolds which are Eilenberg-Maclane spaces. These $K$-theoretic results have now been generalized to $C^{*}$-dynamical systems in $\mathrm{Ma}$.

One of the most outstanding open problems about magnetic Schrodinger operators or Hamiltonians on Euclidean space is concerned with the nature of their spectrum, and is called the Ten Martini Problem (TMP) (cf. Sh). More precisely, TMP asks whether given a multiplier $\sigma$ on $\mathbb{Z}^{2}$, is there an associated Hamiltonian (i.e. a Hamiltonian which commutes with the $(\Gamma, \sigma)$ projective action of $\Gamma$ on $\left.L^{2}\left(\mathbb{R}^{2}\right)\right)$ possessing a Cantor set type spectrum, in the sense that the intersection of the spectrum of the Hamiltonian with some compact interval in $\mathbb{R}$ is a Cantor set? One can deduce from the range of the trace on $K_{0}$ of the twisted group $C^{*}$-algebras that when the multiplier takes its values in the roots of unity in $U(1)$ (we say then that it is rational) that such a Hamiltonian cannot exist. However, in the Euclidean case and for Liouville numbers, the discrete analogue of the TMP has been been settled in the affirmative by Choi, Elliot and Yui [CEY] (cf. Sh] for a historical perspective). In Section 9 we are concerned also with the hyperbolic analogue of the TMP, which we call the Ten Dry Martini Problem (TDMP). We prove that the Kadison constant of the twisted group $C^{*}$-algebra $C_{r}^{*}\left(\Gamma_{g}, \sigma\right)$ is positive whenever the multiplier is rational, where $\Gamma_{g}$ is now the fundamental group of a genus $g$ Riemann surface. We then use the results of Brüning and Sunada $\mathrm{BrSu}$ to deduce that when the multiplier is rational the TDMP answered in the negative, and we leave open the more difficult irrational case. The calculation of the range of the trace exploits a number of results including a twisted Kasparov map on $K$-theory. Finally, we apply our results to give a complete classification up to isomorphism of the twisted $C^{*}$-algebras $C_{r}^{*}\left(\Gamma_{g}, \sigma\right)$. 
In Sections 10 and 11 we will identify the character of our Fredholm modules, the 'Hall conductivity', for both the continuous and discrete models. This character $\tau_{c}(P, P, P)=$ $\operatorname{tr}(P d P d P)$ is shown to arise from Connes' 'area cocycle' and we are able to identify it with a topological invariant, generalising the work of Xia [Xia] in the case of the quantum Hall effect on Euclidean space. We use the pairing between $K$-theory and cyclic cohomology [Co], a generalization of the Connes-Moscovici index theorem [CM] to projectively invariant elliptic operators and the twisted analogue of the Kasparov map. In fact we obtain a general index theorem which equates the (analytical) index arising from the Fredholm modules to a topological index. It specialises in the case of the cyclic cocycle $\tau_{c}$ to give the surprising fact that the hyperbolic 'Hall conductivity' $\tau_{c}(P, P, P) \in 2(g-1) \mathbb{Z}$. This raises the obvious question of whether a real material with a hyperbolic crystalline geometry could be manufactured and the genus of the quotient Riemann surface measured experimentally. To be specific the model we consider here can be understood most easily in the imbedded hyperboloid version of hyperbolic space. If we use the $\Gamma$-orbit of a point in the hyperboloid in $\mathbb{R}^{3}$ to represent the crystal lattice structure of a conducting material then our discrete model corresponds to applying a magnetic field which is everywhere normal to the hyperboloid. (This captures the hyperbolic geometry.) Then by regarding the lattice points as the vertices of a graph whose edges are geodesics corresponding to the generators of $\Gamma$ our model Hamiltonian corresponds to allowing electrons to hop between sites on the lattice along the edges of the graph. Then our theorem predicts that the conductivity should depend on the genus of the Riemann surface obtained by quotienting the hyperboloid by $\Gamma$.

In Section 6 we exhibit a cyclic cocycle which plays the role of the Kubo formula for higher genus surfaces. It has an intrinsic geometric description as a 'symplectic area' cocycle on the universal cover of the Jacobi variety of the Riemann surface. The novel feature of the higher genus case (as opposed to genus one which is the Euclidean case) is that the Kubo cocycle is cohomologous (but not equal) to the cyclic cocycle arising from the Fredholm module. Given our $K$-theoretic interpretation of the latter this is sufficient to give the anticipated result that the Hall conductivity, as defined through the Kubo cocycle, is integral and depends on the genus.

We conclude by showing how our formalism links with the non-commutative Riemann surface theory described in Klim+Les], Klim+Les2].

\section{THE GEOMETRY OF THE HYPERBOLIC PLANE}

The upper half-plane can be mapped by the Cayley transform $z=(\zeta-i) /(\zeta+i)$ to the unit disc $\mathbf{D}$ equipped with the metric $|d z|^{2} /\left(1-|z|^{2}\right)^{2}$ and symplectic form $d z d \bar{z} / 2 i(1-$ $\left.|z|^{2}\right)^{2}$, on which $\mathbf{S U}(1,1)$ acts, and some calculations are more easily done in that setting. In order to preserve flexibility we shall work more abstractly with a Lie group $G$ acting transitively on a space $X \sim G / K$. Although we shall ultimately be interested in the case of $G=\mathbf{S L}(2, \mathbb{R})$ or $\mathbf{S U}(1,1)$, and $K$ the maximal compact subgroup which stabilises $\zeta=i$ or $z=0$, those details will play little role in many of our calculations, though we shall need to assume that $X$ has a $G$-invariant Riemannian metric and symplectic form $\omega_{\mathbb{H}}$. We 
shall denote by $\Gamma$ a discrete subgroup of $G$ which acts freely on $X$ and hence intersects $K$ trivially.

We shall assume that $\mathcal{L}$ is a hermitian line bundle over $X$, with a connection, $\nabla$, or equivalently, for each pair of points $w$ and $z$ in $X$, we denote by $\tau(z, w)$ the parallel transport operator along the geodesic from $\mathcal{L}_{w}$ to $\mathcal{L}_{z}$. In $\mathbb{H}$ with the line bundle trivialised and $\eta=\theta d x / y$ one can calculate explicitly that

$$
\tau(z, w)=\exp \left(i \int_{w}^{z} \eta\right)=[(z-\bar{w}) /(w-\bar{z})]^{\theta} .
$$

For general $\eta$ we have $\eta-\theta d x / y=d \phi$ and

$$
\tau(z, w)=\exp \left(i \int_{w}^{z} \eta\right)=[(z-\bar{w}) /(w-\bar{z})]^{\theta} \exp (i(\phi(z)-\phi(w))) .
$$

Parallel transport round a geodesic triangle with vertices $z, w, v$, gives rise to a holonomy factor:

$$
\varpi(v, w, z)=\tau(v, z)^{-1} \tau(v, w) \tau(w, z),
$$

and this is clearly the same for any other choice of $\eta$, so we may as well work in the general case.

Lemma 1. The holonomy can be written as $\varpi(v, w, z)=\exp \left(i \theta \int_{\Delta} \omega_{\mathbb{H}}\right)$, where $\Delta$ denotes the geodesic triangle with vertices $z, w$ and $v$. The holonomy is invariant under the action of $G$, that is $\varpi(v, w, z)=\varpi(g v, g w, g z)$, and under cyclic permutations of its arguments. Transposition of any two vertices inverts $\varpi$. For any four points $u, v, w, z$ in $X$ one has

$$
\varpi(u, v, w) \varpi(u, w, z)=\varpi(u, v, z) \varpi(v, w, z) .
$$

Proof. By definition, for a suitable trivialisation of $\mathcal{L}$ one has

$$
\varpi(v, w, z)=\exp \left(i \int_{\partial \Delta} \eta\right)
$$

and the first part follows by applying Stokes' Theorem after noting that the result is independent of the trivialisation. The invariance under $G$ follows from the invariance of the symplectic form, and the results of permutations follow from the properties of the integral, as does the final identity.

\section{The TWISTED ALGEBRA OF KERNELS}

The geometrical data described in the last section enables us to easily describe the first of the two $\mathrm{C}^{*}$ algebras which appear in the theory. This twisted algebra of kernels, which was introduced by Connes [Co2 is the $\mathrm{C}^{*}$-algebra $\mathcal{B}$ generated by compactly supported smooth functions on $X \times X$ with the multiplication

$$
k_{1} * k_{2}(z, w)=\int_{X} k_{1}(z, v) k_{2}(v, w) \varpi(z, w, v) d v
$$

(where $d v$ denotes the $G$-invariant measure defined by the metric) and $k^{*}(z, w)=\overline{k(w, z)}$. There is an obvious trace on $\mathcal{B}$ given by $\tau_{\mathcal{B}}(k)=\int_{X} k(z, z) d z$ The algebra of twisted 
kernels is the extension of the $\mathrm{C}^{*}$-algebra of the principal groupoid $X \times X$ defined by the cocycle $((v, w),(w, z)) \mapsto \varpi(v, w, z)$, [Ren1].

Lemma 2. The algebra $\mathcal{B}$ has a representation $\pi$ on the space of $L^{2}$ sections of $\mathcal{L}$ defined by

$$
(\pi(k) \psi)(z)=\int_{X} k(z, w) \tau(z, w) \psi(w) d w
$$

Proof. The parallel transport $\tau(z, w)$ ensures that the integral is in the appropriate fibre, and the fact that it is a representation follows from a calculation using the definition of the holonomy.

Before describing the second algebra we need to link the geometrical data more directly to the group $G$. To do this we fix a basepoint $u \in X$ and introduce the function $\phi$ from $X \times G$ to line bundle automorphisms defined by

$$
\phi(z, g)=\varpi\left(u, g^{-1} u, g^{-1} z\right) \tau(u, z)^{-1} \tau\left(u, g^{-1} z\right) .
$$

(The ratio of parallel transports defines an operator from the fibre $\mathcal{L}_{g^{-1} z}$ to $\mathcal{L}_{z}$.)

Lemma 3. The function $\phi$ satisfies

$$
\begin{aligned}
\phi(z, x) \phi\left(x^{-1} z, y\right) & =\varpi\left(u, y^{-1} u, y^{-1} x^{-1} u\right) \phi(z, x y) \\
\phi(z, x) \tau\left(x^{-1} z, x^{-1} w\right) & =\tau(z, w) \phi(w, x) .
\end{aligned}
$$

Proof. By definition we have

$$
\begin{aligned}
& \phi(z, x) \phi\left(x^{-1} z, y\right)=\varpi\left(u, x^{-1} u, x^{-1} z\right) \varpi\left(u, y^{-1} u, y^{-1} x^{-1} z\right) \tau(u, z)^{-1} \\
& \tau\left(u, x^{-1} z\right) \tau\left(u, x^{-1} z\right)^{-1} \tau\left(u, y^{-1} x^{-1} z\right) \\
&=\frac{\varpi\left(u, x^{-1} u, x^{-1} z\right) \varpi\left(u, y^{-1} u, y^{-1} x^{-1} z\right)}{\varpi\left(u, y^{-1} x^{-1} u, y^{-1} x^{-1} z\right)} \phi(z, x y) .
\end{aligned}
$$

Now by Lemma 2.1

$$
\begin{aligned}
\varpi\left(u, x^{-1} u, x^{-1} z\right) \varpi\left(u, y^{-1} u\right. & \left.y^{-1} x^{-1} z\right) \\
& =\varpi\left(y^{-1} u, y^{-1} x^{-1} u, y^{-1} x^{-1} z\right) \varpi\left(u, y^{-1} u, y^{-1} x^{-1} z\right) \\
& =\varpi\left(u, y^{-1} x^{-1} u, y^{-1} x^{-1} z\right) \varpi\left(u, y^{-1} u, y^{-1} x^{-1} u\right),
\end{aligned}
$$

from which the first result follows. For the second result we note (compressing the notation) that

$$
\begin{aligned}
\frac{\tau\left(x^{-1} z, x^{-1} w\right) \phi(z, x)}{\phi(w, x)} & =\frac{\varpi\left(u, x^{-1} u, x^{-1} z\right)}{\varpi\left(u, x^{-1} u, x^{-1} w\right)} \frac{\tau\left(x^{-1} z, x^{-1} w\right) \tau\left(u, x^{-1} z\right)}{\tau(u, z)} \frac{\tau(u, w)}{\tau\left(u, x^{-1} w\right)} \\
& =\frac{\varpi\left(u, x^{-1} u, x^{-1} z\right) \varpi\left(u, x^{-1} z, x^{-1} w\right)}{\varpi\left(u, x^{-1} u, x^{-1} w\right)} \frac{\tau\left(u, x^{-1} w\right)}{\tau(u, z)} \frac{\tau(u, w)}{\tau\left(u, x^{-1} w\right)} \\
& =\varpi\left(x^{-1} u, x^{-1} z, x^{-1} w\right) \frac{\tau(u, w)}{\tau(u, z)} \\
& =\varpi(u, z, w) \frac{\tau(u, w)}{\tau(u, z)}=\tau(z, w) .
\end{aligned}
$$


The most important aspect of the first result is that

$$
\sigma(x, y)=\phi(z, x y) / \phi(z, x) \phi\left(x^{-1} z, y\right)=\varpi\left(u, y^{-1} u, y^{-1} x^{-1} u\right)^{-1}=\varpi(u, x u, x y u)
$$

is independent of $z$. (We note also that $\sigma(g, 1)=\sigma(1, g)=\sigma\left(g, g^{-1}\right)=1$. Although these normalisations do not seriously affect matters they can sometimes be used to simplify formulae.)

Lemma 4. The function $\sigma: G \times G \rightarrow \mathbb{T}$ satisfies the cocycle identity,

$$
\sigma(x, y) \sigma(x y, g)=\sigma(x, y g) \sigma(y, g) .
$$

Proof. This is a simple calculation along the lines of those above.

This result means that $\sigma$ defines a projective multiplier or group 2-cocycle for $G$, moreover, it is clearly continuous and identically 1 when restricted to $G \times K$ and to $K \times G$.

Lemma 5. The group $G$ has a natural unitary $\sigma$-representation $U$ on the $L^{2}$ sections of $\mathcal{L}$ defined by

$$
U(g) \psi(z)=\phi(z, g) \psi\left(g^{-1} z\right)
$$

Proof. This follows immediately from lemma 3.4.

This projective representation induces an action of $G$ as automorphisms of $\mathcal{B}$.

Lemma 6. For any $g \in G$ and $k \in \mathcal{B}$ we have $U(g) \pi(k) U(g)^{-1}=\pi(g . k)$, where

$$
g . k(z, w)=k\left(g^{-1} z, g^{-1} w\right) .
$$

Proof. By direct calculation and use of Lemma 3.2

$$
\begin{aligned}
\left(U(g) \pi(k) U(g)^{-1} \psi\right)(z) & =\int \phi(z, g) k\left(g^{-1} z, g^{-1} w\right) \tau\left(g^{-1} z, g^{-1} w\right) \phi(w, g)^{-1} \psi\left(g^{-1} w\right) d w \\
& =\int k\left(g^{-1} z, g^{-1} w\right) \tau(z, w) \psi\left(g^{-1} w\right) d w
\end{aligned}
$$

from which the result follows.

The second part of Lemma 3.2 can now be interpreted as saying that the parallel transport $\tau$ behaves covariantly under $U(g)$, that is conjugation by $U(g)$ sends $\tau(z, w)$ to $\tau\left(g^{-1} z, g^{-1} w\right)$. Taking $w=\exp (-t X) z$ and considering the limit as $t \rightarrow 0$ we obtain the following result:

Corollary 1. The $\sigma$-representation $U$ and connection $\nabla$ are related by $U(g) \nabla U(g)^{-1}=$ $g . \nabla$, where $g . \nabla$ denotes the natural action of $G$ on forms. 


\section{VARIOUS $C^{*}$-ALGEBRAS}

4.1. The imprimitivity algebra. The $\sigma$-representation $U$ defined in the previous section is clearly equivalent to one induced from a $\sigma$-representation, $L$, of the isotropy subgroup $K$. Such representations are characterised by the fact that they also admit an action of the imprimitivity algebra. In general this can be defined as one of Green's twisted crossed product $\mathrm{C}^{*}$-algebras [Green], but in the case of a continuous multiplier $\sigma$ there is a simpler direct construction. The imprimitivity algebra, $\mathcal{A}=\mathcal{A}(G, K, \sigma)$, on $G / K$ is a completion of the algebra $\mathcal{A}_{0}=C_{c}(G / K, G)$ with multiplication

$$
(\alpha * \beta)(s, g)=\int_{G} \alpha(s, x) \beta\left(x^{-1} s, x^{-1} g\right) \sigma\left(x, x^{-1} g\right)^{-1} d x
$$

and involution

$$
\alpha^{*}(s, g)=\sigma\left(g, g^{-1}\right) \overline{\alpha\left(g^{-1} s, g^{-1}\right)} .
$$

(With the conventions of the last section $\sigma\left(g, g^{-1}\right)=1$ and could be omitted.) These formulae use the unimodularity of $G$ and the existence of a $G$-invariant measure on $G / K$, otherwise some Radon-Nikodym derivatives would be needed.

The algebra has a trace

$$
\operatorname{tr}_{\mathcal{A}}(\alpha)=\int_{G / K} \alpha(s, 1) d s .
$$

More details may be found in Green, where it is also shown that $\mathcal{A}(G, K, \sigma)$ is Morita equivalent to $C^{*}(K, \sigma) \otimes \mathcal{K}\left(L^{2}(G / K)\right)$, where $C^{*}(K, \sigma)$ denotes the twisted group $C^{*}$ algebra. Like $\mathcal{B}$ the imprimitivity algebra is a groupoid algebra (being an extension by $\sigma$ of the algebra of the transformation groupoid for $G$ acting on $X$ ) and most of this paper could be understood in the context of groupoids, Ren1.

The algebra $\mathcal{B}$ could have been derived from $\mathcal{A}$ as a quotient, as we shall now show. Let $L$ be a $\sigma$-representation of $K$ on a Hilbert space $\mathcal{H}_{L}$. (Since the multiplier of the last section is 1 whenever either of its arguments is in $K$, one could take $L=1$, but the argument works more generally.) For each $L$ the imprimitivity algebra has a natural *representation on the induced representation space of $\mathcal{H}_{L}$-valued functions on $G$ satisfying the equivariance condition

$$
\psi(g k)=\sigma(g, k)^{-1} L(k)^{*} \psi(g)
$$

for all $g \in G$ and $k \in K$. This representation is given by

$$
(\alpha . \psi)(z)=\int_{G} \alpha(z K, x) \sigma\left(x, x^{-1} z\right)^{-1} \psi\left(x^{-1} z\right) d x .
$$

(It may be checked that $\alpha . \psi$ satisfies the same equivariance condition as $\psi$.) The group $G$ has an induced $\sigma$-representation on this function space given by

$$
U(g) \psi(z)=\sigma\left(g, g^{-1} z\right)^{-1} \psi\left(g^{-1} z\right) .
$$

The imprimitivity algebra incorporates both this action and the multiplication operators, and so permits the description of quantum mechanical momentum and position operators on $G / K$. The group action allows for the free Hamiltonian $-\frac{1}{8} \mathbf{J} . \mathbf{J}$, whilst the functions on $X=G / K$ make it possible to add an extra potential, $V$. 
For an appropriate choice of $L, U$ is equivalent to the representation in the last section. Indeed we may identify the equivariant functions on $G$ with sections of the line bundle $\mathcal{L}$ and then we have, in the previous notation,

$$
(\alpha . \psi)(z)=\int_{G} \alpha(z K, x) \phi(z K, x) \psi\left(x^{-1} z\right) d x .
$$

In this form we may easily see the connection to the algebra $\mathcal{B}$ of twisted kernels.

Lemma 7. There is a *-homomorphism $\alpha \mapsto T_{\alpha}$, defined by

$$
T_{\alpha}(z, w)=\int_{G} \frac{\alpha(z, x) \phi(z, x)}{\tau(z, w)} \delta\left(w, x^{-1} z\right) d x
$$

from $\mathcal{A}$ onto $\mathcal{B}$ (where $\delta$ just restricts the integration to those $x$ satisfying $w=x^{-1} z$ ).

Proof. We calculate that

$$
\begin{aligned}
\left(T_{\alpha} * T_{\beta}\right)(z, w) \\
=\int T_{\alpha}(z, v) T_{\beta}(v, w) \frac{\varpi(z, v, w)}{\tau(z, v) \tau(v, w)} d v \\
=\int \alpha(z, x) \beta\left(v, x^{-1} y\right) \frac{\phi(z, x) \phi\left(v, x^{-1} y\right)}{\tau(z, w)} \delta\left(v, x^{-1} z\right) \delta\left(w, y^{-1} x z\right) d x d y d v \\
=\int \alpha(z, x) \beta\left(x^{-1} z, x^{-1} y\right) \frac{\phi(z, x) \phi\left(x^{-1} z, x^{-1} y\right)}{\tau(z, w)} \delta\left(v, x^{-1} z\right) \delta\left(w, y^{-1} z\right) d x d y d v \\
=\int \alpha(z, x) \beta\left(x^{-1} z, x^{-1} y\right) \frac{\phi(z, y)}{\sigma\left(x, x^{-1} y\right) \tau(z, w)} \delta\left(v, x^{-1} z\right) \delta\left(w, y^{-1} z\right) d x d y d v \\
=\int(\alpha * \beta)(z, y) \frac{\phi(z, y)}{\tau(z, w)} \delta\left(w, y^{-1} z\right) d y=T_{\alpha * \beta}(z, w),
\end{aligned}
$$

which proves the homomorphism property. It follows similarly that it is a $*$-homomorphism.

To see that it is surjective we note that if the Haar measure on $K$ is normalised then

$$
\alpha(z, x)=T_{\alpha}\left(z, x^{-1} z\right) \frac{\tau\left(z, x^{-1} z\right)}{\phi(z, x)}
$$

provides an inverse. (The key is to note that if $g(z) u=z$ and $g(w) u=w$ then $w=x^{-1} z$ forces $x$ to have the form $g(z) k g(w)^{-1}$ for some $k \in K$.)

This may be interpreted as saying that $\mathcal{B}$ is a quotient of $\mathcal{A}$, and this means that the behaviour of the two algebras is very similar. For this reason we shall often merely show the constructions in the case of one and leave it as an exercise to fill in the details for the other. However, it will be useful to note that $\mathcal{B}$ has a natural trace

$$
\operatorname{tr}_{\mathcal{B}}(T)=\int_{X} T(z, z) d z .
$$


4.2. The regular representation. The trace gives rise to an inner product

$$
\langle\alpha, \beta\rangle=\operatorname{tr}_{\mathcal{A}}\left(\alpha^{*} * \beta\right)=\int_{G / K \times G} \overline{\alpha(s, x)} \beta(s, x) d s d x
$$

on $\mathcal{A}_{0}$, and completion with respect to this gives rise to a Hilbert space $\mathcal{H}_{\mathcal{A}}$ on which $\mathcal{A}_{0}$ is represented by left multiplication. Since the action is continuous this extends to give the left regular representation of $\mathcal{A}$. This is given by the same formula as the algebra multiplication. That is, for $\Psi \in \mathcal{H}_{\mathcal{A}}$, one has

$$
(\alpha . \psi)(s, g)=\int_{G} \alpha(s, x) \psi\left(x^{-1} s, x^{-1} g\right) \sigma\left(x, x^{-1} g\right)^{-1} d x .
$$

There is also a $\sigma$-representation of $G$ on $\mathcal{H}_{\mathcal{A}}$, obtained by sending $g \in G$ to the function $(s, x) \mapsto \delta_{g}(x)$.

For any unitary character $\chi$ of $K$, one may define a generalised regular representation on the space $H_{\mathcal{A}}^{\chi}$ of functions $\psi \in C_{c}(G \times G)$ which satisfy the condition

$$
\psi(z k, g)=\chi(k) \psi(z, g) .
$$

Since $|\psi(z k, g)|^{2}$ is independent of $k$, we may use the same inner product as before, and it is easy to check that the action

$$
(\alpha . \psi)(z, g)=\int_{G} \alpha(z K, x) \psi\left(x^{-1} z, x^{-1} g\right) \sigma\left(x, x^{-1} g\right)^{-1} d x
$$

respects the equivariance condition. When $\chi=1$ we obtain the regular representation.

We could similarly define the regular representation of $\mathcal{B}$ and also a generalisation defined by

$$
(T . \Psi)(z, w)=\int_{X} T(z K, v) \Psi(v, w) d v
$$

on the space of kernels $\Psi$ on $G \times G$ which satisfy $\Psi\left(z k_{1}, w k_{2}\right)=\chi\left(k_{2}\right) \Psi(z, w)$.

4.3. The $\Gamma$-invariant imprimitivity algebra. Let $\Gamma$ be another subgroup of $G$, and let $\mathcal{A}^{\Gamma}$ denote the part of the imprimitivity algebra which commutes with the induced representation of $\Gamma$. This is readily seen to consist of those functions $\alpha \in \mathcal{A}(G, K, \sigma)$ which satisfy the condition $\gamma \cdot \alpha=\alpha$, where

$$
(\gamma \cdot \alpha)(s, g)=\sigma(g, \gamma) \sigma\left(\gamma, \gamma^{-1} g \gamma\right)^{-1} \alpha\left(\gamma^{-1} s, \gamma^{-1} g \gamma\right)
$$

for all $\gamma \in \Gamma$. To see this we note that

$$
\left(\delta_{\gamma} * \alpha\right)(s, g)=\sigma\left(\gamma, \gamma^{-1} g\right) \alpha\left(\gamma^{-1} s, \gamma^{-1} g\right),
$$

which is identical to

$$
\left(\gamma . \alpha * \delta_{\gamma}\right)(s, g)=\sigma\left(g \gamma^{-1}, \gamma\right)^{-1} \gamma \cdot \alpha\left(s, g \gamma^{-1}\right)=\sigma\left(\gamma, \gamma^{-1} g\right)^{-1} \alpha\left(\gamma^{-1} s, \gamma^{-1} g\right) .
$$

When $g=1$ the condition $\gamma \cdot \alpha(s, g)=\alpha(s, g)$ reduces to $\alpha\left(\gamma^{-1} s, 1\right)=\alpha(s, 1)$ so that one obtains a trace

$$
\operatorname{tr}_{\mathcal{A}^{\Gamma}}(\alpha)=\int_{\Gamma \backslash G / K} \alpha(s, 1) d s
$$


(More generally, the $\Gamma$-invariant functions are determined by their values at a single point $s$ of each $\Gamma$ orbit on $G / K$.) Using $\operatorname{tr}_{\mathcal{A}^{\Gamma}}$ one may define a regular representation of $\mathcal{A}^{\Gamma}$.

In the case of the twisted kernel algebra one may likewise pick out a $\Gamma$-invariant subalgebra $\mathcal{B}^{\Gamma}$, which commutes with $U(\gamma)$ for all $\gamma \in \Gamma$. Using Lemma 3.2 this condition reduces simply to the requirement that the kernel satisfies $k\left(\gamma^{-1} z, \gamma^{-1} w\right)=k(z, w)$. The natural trace $\operatorname{tr}_{\mathcal{B}}$ Г for this algebra is given by the same formula as before except that the integration is over a fundamental domain $X_{\Gamma}$ rather than $X$ :

$$
\tau_{\mathcal{B}^{\Gamma}}(T)=\int_{X_{\Gamma}} T(z, z) d z .
$$

4.4. Morita equivalence. Later we shall need some $K$-theory, and so it will be useful to show that the algebra $\mathcal{B}^{\Gamma}$ is Morita equivalent to another more tractable algebra. We shall do this by using the groupoid equivalence arguments of $\mathrm{M}+\mathrm{R}+\mathrm{W}$, or rather the twisted version, Ren2], Ren3]. We have already noted that $\mathcal{B}$ is an extension of the groupoid $X \times X$ by a cocycle defined by $\varpi$, and $\Gamma$ invariance of $\varpi$ means that $\mathcal{B}^{\Gamma}$ is likewise the extension of $X \times_{\Gamma} X$ by $\varpi$, where $X \times_{\Gamma} X$ denotes the groupoid obtained by factoring out the diagonal action of $\Gamma$. More precisely, the groupoid elements are $\Gamma$ orbits $(x, y)_{\Gamma}=\{(\gamma x, \gamma y): \gamma \in \Gamma\}$, and $\left(x_{1}, y_{1}\right)_{\Gamma}$ and $\left(x_{2}, y_{2}\right)_{\Gamma}$ are composable if and only if $y_{1}=\gamma x_{2}$ for some $\gamma \in \Gamma$, and then the composition is $\left(x_{1}, \gamma y_{2}\right)_{\Gamma}$.

Theorem 1. The algebra $\mathcal{B}^{\Gamma}$ is Morita equivalent to the twisted group algebra $C^{*}(\Gamma, \bar{\sigma})$.

Proof. This result will follow immediately from [Ren2] Corollaire 5.4 (cf [M+R+W] Theorem 2.8) once we have established the groupoid equivalence in the following Lemma.

Lemma 8. The line bundle $\mathcal{L}$ over $X$ provides an equivalence (in the sense of Ren2] Definition 5.3) between the groupoid extensions $\left(X \times_{\Gamma} X\right)^{\varpi}$ of $X \times_{\Gamma} X$ defined by $\varpi$ and $\Gamma^{\sigma}$ of $\Gamma$ defined by $\bar{\sigma}$.

Proof. Both extensions are by $\mathbb{T}$. We write the elements of $\left(X \times_{\Gamma} X\right)^{\varpi}$ as triples $(x, y, t) \in$ $X \times X \times \mathbb{T}$ with the first two elements representing a diagonal $\Gamma$ orbit. Elements are composable if their first two components are composable, and, when $y_{1}=\gamma x_{2}$,

$$
\left(x_{1}, y_{1}, t_{1}\right)\left(x_{2}, y_{2}, t_{2}\right)=\left(x_{1}, \gamma y_{2}, t_{1} t_{2} \varpi\left(x_{1}, y_{1}, \gamma y_{2}\right)\right) \text {. }
$$

The line bundle can be trivialised and written as $X \times \mathbb{C}$. We let $\left(X \times_{\Gamma} X\right)^{\varpi}$ act on the left of the line bundle by defining $(x, y, t)$ to act on $(z, u)$ if $z=\gamma y$ for some $\gamma \in \Gamma$, and then the result of the action is $(\gamma x, \tau(\gamma x, z) t u$ ). (One may check that this gives an action using the relationship between parallel transport and holonomy and the $\Gamma$-invariance of $\varpi$.

The twisted groupoid $\Gamma^{\sigma}$ has underlying set $\Gamma \times \mathbb{T}$, all elements are composable, and multiplication is given by

$$
(\beta, s)(\gamma, t)=(\beta \gamma, \sigma(\beta, \gamma) s t)
$$


It acts on the right of $\mathcal{L}$ by

$$
(z, u) \cdot(\gamma, t)=\left(\gamma^{-1} z, \phi(z, \gamma)^{-1} t u\right)
$$

(The fact that this defines an action follows from the definition of $\sigma$ in terms of $\phi$.) We may now check that these actions commute, since, if $z=\beta y$,

$$
[(x, y, t)(z, u)](\gamma, s)=(\beta x, \tau(\beta x, z) t u)(\gamma, s)=\left(\gamma^{-1} \beta x, \phi(\beta x, \gamma)^{-1} \tau(\beta x, z) t u s\right),
$$

whilst

$$
(x, y, t)[(z, u)(\gamma, s)]=(x, y, t)\left(\gamma^{-1} z, \phi(z, \gamma)^{-1} u s\right)=\left(\gamma^{-1} \beta x, \tau\left(\gamma^{-1} \beta x, \gamma^{-1} z\right) \phi(z, \gamma)^{-1} t u s\right) \text {, }
$$

and the equality of these two follows from the second part of Lemma 3.2.

Remarks. We will observe in Section 9 that the algebra $C^{*}(\Gamma, \bar{\sigma})$ is isomorphic to $C^{*}(\Gamma, \sigma)$ which in turn is known to be isomorphic to the imprimitivity algebra for $\sigma$-inducing from $\Gamma$ to $C^{*}(G, \sigma)$. This latter algebra, denoted $C^{*}(G / \Gamma, G)$ is the completion of $C_{c}(G / \Gamma, G)$ where the latter has a multiplication analogous to that described above for $C_{c}(G / K, G)$ (simply replace $K$ by $\Gamma$ in the earlier discussion). Thus a corollary of our results in this subsection is that $\mathcal{B}^{\Gamma}$ is Morita equivalent to $C^{*}(G / \Gamma, G)$. Furthermore our discussion below of a Fredholm module for $\mathcal{B}^{\Gamma}$ may be modified so as to produce a Fredholm module for $C^{*}(G / \Gamma, G)$ whose character, for $G=\mathbf{S O}(n, 1)$, is also given by the area cocycle. We omit the details here as they would take us too far afield (see however the analogous discussion in the discrete case in [Co2]).

4.5. The Hamiltonian. We have asserted informally that the Hamiltonian can be accommodated within the algebras $\mathcal{A}^{\Gamma}$ and $\mathcal{B}^{\Gamma}$ and we shall now provide the proof. We work with the smaller algebra $\mathcal{B}^{\Gamma}$, the results for $\mathcal{A}^{\Gamma}$ following similarly.

Lemma 9. The Hamiltonian $H=-\frac{1}{2} \nabla^{*} . \nabla$ commutes with the projective representation $U$.

Proof. We recall from Corollary 3.6 that $U(g) \nabla U(g)^{-1}=g . \nabla$, so that

$$
U(g) H U(g)^{-1}=-\frac{1}{2}(g \cdot \nabla)^{*}(g \cdot \nabla),
$$

and, since the Riemannian structure is invariant under the action of $G$, this is just $H$.

We could also obtain the same result by writing $H=\frac{1}{8} \mathbf{J} . \mathbf{J}+\frac{1}{4} B^{2}$ (for some constant $B$ ) and using the fact that the Casimir operator commutes with the representation, provided that we check that $J_{k}$ are the representatives of the Lie algebra generators in the representation $U$. Using the invariance of $H$ it is now not difficult to see the following result:

Lemma 10. The Hamiltonian $H$ is affiliated to the von Neumann algebra generated by the representation $\pi$ of $\mathcal{B}$.

Since $H$ is $G$-invariant and so a fortiori also $\Gamma$-invariant, it is sufficient to look at $\mathcal{B}^{\Gamma}$. 
Corollary 2. The Hamiltonian $H$ is affiliated to the von Neumann algebra generated by the representation $\pi$ of $\mathcal{B}^{\Gamma}$.

We next observe that Brüning and Sunada have proved an estimate on the Schwartz kernel of $\exp (-t H)$ for $t>0$, which implies that it is $L^{1}$ in each variable separately. Since this kernel is $\Gamma$-invariant (by Lemma 7.1) it follows (in exactly the same fashion as Lemma 4 of $[\mathrm{BrSu}]$ ) that this estimate implies that $\exp (-t H)$ is actually in the algebra $\mathcal{B}^{\Gamma}$.

Lemma 11. The operator $e^{-t H}$ is an element of $\mathcal{B}^{\Gamma}$.

Corollary 3. The spectral projections of $H$ corresponding to gaps in the spectrum lie in $\mathcal{B}^{\Gamma}$.

Proof. If $\mu$ lies in a gap of the spectrum of $H$ then let $f$ be a continuous approximate step function which is identically one on the part of the spectrum of $H$ contained in $[0, \mu]$ and zero on the part contained in $[\mu, \infty)$. Define $g(x)=f \circ \ln (1 / x)$ for $x \in[0,1]$. Then $g$ is a bounded continuous function which, when applied to $e^{-t H}$ gives the spectral projection corresponding to the interval $[0, \mu]$.

Finally we consider the interacting Hamiltonian $H+V$, where $V$ is a $\Gamma$-invariant function on $X$. Notice that if $\psi$ is a continuous function of compact support on $(X \times X) / \Gamma$ then $V \psi$ is also such a function and hence defines an element in the groupoid algebra. Now, by Lemma 11, the resolvent of $H$ lies in $\mathcal{B}^{\Gamma}$ and by writing

$$
(z-H-V)^{-1}=\left(1-(z-H)^{-1} V\right)^{-1}(z-H)^{-1},
$$

and expanding $\left(1-(z-H)^{-1} V\right)^{-1}$ in a power series, we see that the resolvent of $H+V$ is in the algebra $\mathcal{B}^{\Gamma}$. This entails by a simple modification of Corollary 3 that the spectral projections of $H+V$ corresponding to a gap in the spectrum also lie in $\mathcal{B}^{\Gamma}$.

\section{THE DISCRETE MODEL}

In this section we formulate a version of the integer quantum Hall effect on a graph in hyperbolic space. The discussion uses a construction due to Sunada [Sun together with a modification of Connes' work on Fredholm modules for the group $C^{*}$-algebra of a discrete subgroup of a Lie group [Co2].

The graph is obtained by taking our group $\Gamma$, the fundamental group of a Riemann surface, which acts freely on hyperbolic space, fixing a base point $u$ and taking the orbit through $u$ under the $\Gamma$ action. This gives the vertices of the graph. The edges of the graph are geodesics constructed as follows. Each element of the group may be written as a word of minimal length in the $2 g$ generators and their inverses. Each generator and its inverse determines a unique geodesic emanating from a vertex $x$ and these form the edges of the graph. Thus each word $x$ in the generators determines a piecewise geodesic path from $u$ to $x$. 
Sunada constructs a Hamiltonian on $\ell^{2}(\Gamma . u)$ which is a generalised Harper operator. This construction, specialised to our case, has the following form. First we note that we may trivialise the restriction of the line bundle to the vertices and so without loss of generality the appropriate Hilbert space becomes $\ell^{2}(\Gamma . u)$. While the construction works for any connection 1-form $A$ on a line bundle $L$ over hyperbolic space we make the formulae explicit by restricting to the case where $A$ is the one form $\eta$. For each directed edge $e$ of the graph joining $o(e)$ to $t(e)$ we define a function $\tau(e)=\exp \left(i \int_{e} \eta\right)$. Then $\tau(e)$ satisfies

$$
\tau(\gamma . e)=\tau(e)\left(\frac{\overline{c o(e)}+d}{c o(e)+d}\right)^{\theta}\left(\frac{c t(e)+d}{c \overline{t(e)}+d}\right)^{\theta}
$$

where $\gamma=\left(\begin{array}{ll}a & b \\ c & d\end{array}\right)$. We introduce the notation

$$
s_{\gamma}(w)=\left(\frac{c \bar{w}+d}{c w+d}\right)^{\theta} .
$$

Remarks 2. Note that in our earlier notation these definitions amount to

$$
\tau(e) \equiv \tau(t(e), o(e)),
$$

while the function from $\Gamma \times H$ to $U(1)$ given by $(\gamma, w) \mapsto s_{\gamma}(w)$ is a projective 1-cocycle for the $\Gamma$ action on $H$ which is cohomologous to the function mapping $(\gamma, w) \mapsto \phi(\gamma \cdot w, \gamma)$.

Consequently there is a projective action of $\Gamma$ on $\ell^{2}(\Gamma . u)$ given on $f \in \ell^{2}(\Gamma . u)$ by

$$
\tilde{\rho}_{\gamma} f(w)=s_{\gamma}\left(\gamma^{-1} w\right) f\left(\gamma^{-1} w\right)
$$

We have

$$
\tilde{\rho}_{\gamma_{1} \gamma_{2}}=\frac{s_{\gamma_{1} \gamma_{2}}\left(\gamma_{2}^{-1} \gamma_{1}^{-1} x\right)}{s_{\gamma_{1}}\left(\gamma_{1}^{-1} x\right) s_{\gamma_{2}}\left(\gamma_{2}^{-1} \gamma_{1}^{-1} x\right)} \tilde{\rho}_{\gamma_{1}} \tilde{\rho}_{\gamma_{2}},
$$

and Sunada shows that the function multiplying $\tilde{\rho}_{\gamma_{1}} \tilde{\rho}_{\gamma_{2}}$ is independent of $x$. This is exactly the relation found at the end of lemma 3: the correspondence is given by $\phi(z, *) \leftrightarrow$ $s_{*}\left(*^{-1} z\right)$. To obtain an explicit expression for this function, let $\gamma_{3}=\gamma_{1} \gamma_{2}$, and write $\left(\gamma_{i}\right)=\left(\begin{array}{ll}a_{i} & b_{i} \\ c_{i} & d_{i}\end{array}\right)$ for $i=1,2,3$. Then a direct calculation shows that

$$
\tilde{\rho}_{\gamma_{1} \gamma_{2}}=\sigma\left(\gamma_{1}, \gamma_{2}\right) \tilde{\rho}_{\gamma_{1}} \tilde{\rho}_{\gamma_{2}}
$$

where $\sigma$ is given by

$$
\sigma\left(\gamma_{1}, \gamma_{2}\right)=\exp \left(2 i \theta\left(\arg \left(c_{3} \bar{w}+d_{3}\right)-\arg \left(c_{1}\left(\overline{\gamma_{2} w}\right)+d_{1}\right)-\arg \left(c_{2} \bar{w}+d_{2}\right)\right)\right) .
$$

It is not difficult to see that the right hand side is independent of the choice of $w$.

Following [Sun], we define our discrete hamiltonian, for $f \in \ell^{2}(\Gamma . u)$, by

$$
h_{\tau} f(w)=\sum_{\substack{e \\ o(e)=w}} \tau(e) f(t(e)) .
$$

Then $h_{\tau}$ is a generalised difference operator (Sunada shows that the Harper operator arises in a similar fashion). One can verify by direct calculation that $h_{\tau}$ commutes with the projective action of $\Gamma$. This will, however, become readily apparent when we transfer this construction to $\ell^{2}(\Gamma)$. Define $\iota: \ell^{2}(\Gamma . u) \rightarrow \ell^{2}(\Gamma)$ by:

$$
\iota(f)(\gamma)=\tilde{\rho}(\gamma) f(u) .
$$


Observe that

$$
\iota(\tilde{\rho}(\mu) f)(\gamma)=\sigma(\gamma, \mu) \iota(f)(\gamma \mu) .
$$

Thus $\iota$ intertwines $\tilde{\rho}$ with the $\bar{\sigma}$-representation:

$$
\rho(\mu) \iota(f)(\gamma)=\sigma(\gamma, \mu) \iota(f)(\gamma \mu) .
$$

Henceforth we use $\iota$ to identify $\ell^{2}(\Gamma . u)$ with $\ell^{2}(\Gamma)$.

Proposition 1. Sun The operator $h_{\tau}$ on $\ell^{2}(\Gamma . u)$ maps to the operator $H_{\tau}$ on $\ell^{2}(\Gamma)$ under $\iota$, where

$$
H_{\tau} f(\gamma)=\sum_{\mu \in \Gamma} \sigma\left(\mu, \mu^{-1} \gamma\right)^{-1} a(\mu) f\left(\mu^{-1} \gamma\right)
$$

and $a$ is the function on $\Gamma$ given by

$$
a(\gamma)=s_{\gamma}(u) \sum_{\substack{e \\ o(e)=u \\ t(e)=\gamma \cdot u}} \tau(e) .
$$

Corollary 4. The bounded self-adjoint operator $H_{\tau}$ is in the algebraic twisted group algebra $\mathbb{C}(\Gamma, \sigma)$ (the elements of finite support in $C^{*}(\Gamma, \sigma)$ ) as the function a has finite support. Moreover $H_{\tau}$ acts on the left as an element of this algebra and so commutes with the $\bar{\sigma}$-representation $\rho$, as the latter acts on the right.

\section{A Kubo Formula}

6.1. Conductivity cocycles. In this subsection we present an argument which derives analogues of the Kubo formula for the hyperbolic 'Hall conductivity'. In subsection 7.2 we show how to construct, from the results of this subsection, a unique cocycle which may be compared with the character of the Fredholm module of Section 7.

Our reasoning here is that the Hall conductivity in the Euclidean situation is measured experimentally by determining the equilibrium ratio of the current in the direction of the applied electric field to the Hall voltage, which is the potential difference in the orthogonal direction. To calculate this mathematically we instead determine the component of the induced current that is orthogonal to the applied potential. The conductivity can then be obtained by dividing this quantity by the magnitude of the applied field. In the hyperbolic case it would seem at first sight that there are no preferred directions. However interpreting the generators of the fundamental group as geodesics on hyperbolic space gives a family of preferred directions emanating from the base point. For each pair of directions it is therefore natural to imitate the procedure of the Euclidean case and mathematically this is done as follows.

The Hamiltonian $H$ in a magnetic field depends on the magnetic vector potential $\mathbf{A}$ and the functional derivative $\delta_{k} H$ of $H$ with respect to the components of $\mathbf{A}$, denoted $A_{k}$, gives the current density $J_{k}$. (For simplicity we take variations within a one-parameter family.) The expected value of the current in a state described by a projection operator $P$ is therefore $\operatorname{tr}\left(P \delta_{k} H\right)$ (cf $\mathrm{Av}+\mathrm{S}+\mathrm{Y}$ equation (3.2)). The following claim is not proved by a rigorous argument: one needs to check various analytical details as in Xia. We have 
refrained from doing so here as this would take us too far afield from the main point, namely, obtaining a sensible hyperbolic Kubo formula which may be compared with the character of the Fredholm module constructed in the next section. For the moment tr will denote a generic trace. We will become specific after extracting a rigorous definition of the Kubo formula.

\section{Claim:}

$$
\operatorname{tr}\left(P \delta_{k} H\right)=i \operatorname{tr}\left(P\left[\partial_{t} P, \delta_{k} P\right]\right)
$$

Plausability argument: By using the invariance of the trace under the adjoint action of operators and the equation of motion we see that

$$
\begin{aligned}
\operatorname{tr}\left(P\left[\partial_{t} P, \delta_{k} P\right]\right) & =-\operatorname{tr}\left(\left[P, \delta_{k} P\right] \partial_{t} P\right) \\
& =-i \operatorname{tr}\left(\left[P, \delta_{k} P\right][P, H]\right) \\
& =i \operatorname{tr}\left(\left[P,\left[P, \delta_{k} P\right]\right] H\right) .
\end{aligned}
$$

Now $\delta_{k} P=\delta_{k}\left(P^{2}\right)=P\left(\delta_{k} P\right)+\left(\delta_{k} P\right) P$, whence $P\left(\delta_{k} P\right) P=0$ and we have

$$
\begin{aligned}
{\left[P,\left[P, \delta_{k} P\right]\right] } & =P\left(P\left(\delta_{k} P\right)-\left(\delta_{k} P\right) P\right)-\left(P\left(\delta_{k} P\right)-\left(\delta_{k} P\right) P\right) P \\
& =P\left(\delta_{k} P\right)+\left(\delta_{k} P\right) P=\delta_{k} P .
\end{aligned}
$$

Consequently we may write

$$
\operatorname{tr}\left(P\left[\partial_{t} P, \delta_{k} P\right]\right)=i \operatorname{tr}\left(\left(\delta_{k} P\right) H\right)=i \operatorname{tr}\left(\delta_{k}(P H)\right)-i \operatorname{tr}\left(P\left(\delta_{k} H\right)\right),
$$

and, assuming that the trace is invariant under variation of $A_{k}$, the first term vanishes leaving the result asserted.

If the only $t$-dependence in $H$ and $P$ is due to the variation of $A_{j}$, a component distinct from $A_{k}$, then $\partial_{t}=\partial A_{j} / \partial t \times \delta_{j}$. Working in the Landau gauge so that the electrostatic potential vanishes, the electric field is given by $\mathbf{E}=-\partial \mathbf{A} / \partial t$, and so $\partial_{t}=-E_{j} \delta_{j}$. Combining this with the previous argument we arrive at the following result:

Corollary 5. The conductivity for currents in the $k$ direction induced by electric fields in the $j$ direction is given by $-i \operatorname{tr}\left(P\left[\delta_{j} P, \delta_{k} P\right]\right)$.

Proof. The expectation of the current $J_{k}$ is given by

$$
\operatorname{tr}\left(P \delta_{k} H\right)=i \operatorname{tr}\left(P\left[\partial_{t} P, \delta_{k} P\right]\right)=-i E_{j} \operatorname{tr}\left(P\left[\delta_{j} P, \delta_{k} P\right]\right),
$$

from which the result follows immediately.

6.2. The derivations on a Riemann surface. On a Riemann surface it is natural to investigate changes in the potential corresponding to adding multiples of the real and imaginary parts of holomorphic 1-forms. (For the genus 1 torus with imaginary period this amounts to choosing forms whose integral round one sort of cycle vanishes but the integral round the other cycle is non-trivial. This corresponds to putting a non-trivial voltage across one cycle and measuring a current round the other.) 
Let $\Sigma_{g}=\mathbb{H} / \Gamma$ be the Riemann surface determined by quotienting by $\Gamma$. We follow the usual conventions (see for example [GH]) in fixing representative homology generators corresponding to cycles $A_{j}, B_{j}, j=1,2, \ldots, g$ with each pair $A_{j}, B_{j}$ intersecting in a common base point and all other intersection numbers being zero. We let $a_{j}, j=1,2, \ldots, 2 g$ be harmonic 1-forms dual to this homology basis (this means that $a_{j}, j=1, \ldots, g$ are dual to $A_{j}, j=1, \ldots, g$ and $a_{j+g}, j=1,2, \ldots, g$ are dual to $B_{j}, j=1,2, \ldots, g$ ).

Definition. Let

$$
\delta_{j} \tau(z, w)=i \int_{w}^{z} \alpha_{j} \tau(z, w)
$$

and

$$
\delta_{j} \varpi\left(u, g^{-1} u, g^{-1} z\right)=i \int_{\partial \Delta} a_{j} \varpi\left(u, g^{-1} u, g^{-1} z\right)
$$

where $\Delta$ is a triangle with vertices at the three arguments of $\varpi$.

One then calculates that

$$
\begin{aligned}
\delta_{j} \phi(z, g) & =i\left(\int_{\partial \Delta} a_{j}-\int_{u}^{z} a_{j}+\int_{u}^{g^{-1} z} a_{j}\right) \phi(z, g) \\
& =i\left(\int_{u}^{g^{-1} u} a_{j}+\int_{g^{-1} u}^{g^{-1} z} a_{j}-\int_{u}^{z} a_{j}\right) \phi(z, g) .
\end{aligned}
$$

This can also be written as

$$
i \int_{\partial Q} a_{j}-i \int_{g^{-1} z}^{z} a_{j}
$$

where $Q$ denotes the geodesic quadrilateral with vertices at $u, g^{-1} u, g^{-1} z$ and $z$. By Stokes' Theorem the first integral can also be written as $\int_{Q} d a_{j}$, and this vanishes as we chose $a_{j}$ to be the harmonic representative of its class, leaving just

$$
i \int_{g^{-1} z}^{z} a_{j}
$$

Using this last equation in the formula for the action of the imprimitivity algebra (preceding Lemma 7) we see that we have a densely defined derivation on the algebras $\mathcal{A}$ and $\mathcal{B}$ because the action can now be written as the commutator of $\alpha$ with multiplication by the function $\Omega_{j}(z)=i \int_{u}^{z} a_{j}$ :

$$
\delta_{j} \alpha=\left[\Omega_{j}, \alpha\right] .
$$

Suppose that $\alpha$ is a kernel decaying rapidly. By this we mean that it satisfies an estimate

$$
|\alpha(x, y)| \leq \phi(d(x, y))
$$

where $\phi$ is a positive and rapidly decreasing function on $\mathbb{R}$. We claim that $\delta_{j} \alpha$ lies in $\mathcal{A}$ or $\mathcal{B}$ respectively: this follows by noting that the map from $\mathbb{H}$ to $\mathbb{R}^{2 g}$ given by $\Xi: z \mapsto$ $\left(\Omega_{1}(z), \ldots, \Omega_{2 g}(z)\right)$ is the lift to $\mathbb{H}$ of the Jacobi map, $\mathrm{GH}$ (this map is usually regarded as mapping from $\Sigma_{g}$ to the Jacobi variety $J\left(\Sigma_{g}\right)$, however we are thinking of it as a map between the universal covers of these spaces). Now $\Xi$ is globally Lipschitz and this means we may estimate the kernel of $\left[\Omega_{j}, \alpha\right]$. A simple argument shows that it also decays rapidly. 
That this commutator also has the correct properties to define a derivation on $\mathcal{A}^{\Gamma}$ or $\mathcal{B}^{\Gamma}$ follows from the fact that for $\gamma \in \Gamma$,

$$
\Omega_{j}(\gamma . z)-\Omega_{j}(z)
$$

is constant independent of $z$ so that $\Gamma$ equivariance or invariance is preserved. In the case of the torus thought of as a rectangle in $\mathbb{R}^{2}$ with opposite edges identified, one may take $a_{j}+i a_{k}=d z$, and then

$$
\left(\delta_{j}+i \delta_{k}\right) \alpha=i[z-u, \alpha] .
$$

Thus our argument reproduces the standard Kubo formula Xia in the Euclidean case.

Notice that our map $\Xi$ from $\mathbb{H}$ to $\mathbb{R}^{2 g}$ gives the period lattice in $\mathbb{R}^{2 g}$ (that is the lattice determined by the periods of the harmonic forms $a_{j}$ ) to be the standard integer lattice $\mathbb{Z}^{2 g}$ so that $J\left(\Sigma_{g}\right)=\mathbb{R}^{2 g} / \mathbb{Z}^{2 g}$. We may summarise the previous discussion as

Lemma 12. For operators $A_{0}, A_{1}, A_{2}$ in $\mathcal{B}^{\Gamma}$ whose integral kernels are rapidly decaying we have cyclic cocycles defined by

$$
c_{j, k}\left(A_{0}, A_{1}, A_{2}\right)=\operatorname{tr}_{\mathcal{B}^{\Gamma}}\left(A_{0}\left[\delta_{j} A_{1}, \delta_{k} A_{2}\right]\right)=\operatorname{tr}_{\mathcal{B}^{\Gamma}}\left(A_{0}\left[\Omega_{j}, A_{1}\right]\left[\Omega_{k}, A_{2}\right]\right)
$$

for $j, k=1, \ldots, 2 g$.

Each of these formulae for $c_{j k}$ could in principle be regarded as giving a Kubo formula so that we appear to have an embarrassment of riches. However each on their own cannnot be related to the Chern character of the Fredholm module of the next section. A clue as to what is happening is provided by noting that each two form $a_{j} \wedge a_{j+g}$ is harmonic and hence is a multiple of the area two form on $\Sigma_{g}$, thus there is certainly some degeneracy here and we resolve it at the end of the next section.

\section{A Fredholm module}

We shall now assume that $X$ has a spin structure, and we write $\mathcal{S}$ for the spin bundle. The representation of $\mathcal{A}^{\Gamma}$ can then be extended to an action on $\mathcal{H}_{\mathcal{A}}^{\chi} \otimes \mathcal{S}$. This module can be equipped with Fredholm structure by taking $F$ to be Clifford multiplication by a suitable unit vector (to be explained below), and using the product of the trace on $\mathcal{H}_{\mathcal{A}}^{\chi}$ and the graded trace on the Clifford algebra. (If $\varepsilon$ denotes the grading operator on the spinors then the graded trace is just $\operatorname{tr} \circ \varepsilon$.)

The same module can also be described more explicitly: it splits into $\mathcal{H}_{\mathcal{A}}^{\chi} \otimes \mathcal{S}^{+} \oplus \mathcal{H}_{\mathcal{A}}^{\chi} \otimes \mathcal{S}^{-}$ (with the superscripted sign indicating the eigenvalue of $\varepsilon$ ), and this may be written as $\mathcal{H}_{\mathcal{A}}^{\theta_{1}} \oplus \mathcal{H}_{\mathcal{A}}^{\theta_{2}}$. The involution $F$ is then a matrix multiplication operator of the form

$$
F=\left(\begin{array}{cc}
0 & f_{1} \\
f_{2} & 0
\end{array}\right)
$$

with $\left(f_{j} . \psi\right)(z, g)=f_{j}(z, g) \psi(z, g)$ for some suitable functions $f_{j} \in C_{c}(G \times G)$, satisfying $f_{1}=f_{2}^{-1}$. For consistency, we require that for any $\psi \in \mathcal{H}_{\mathcal{A}}^{\theta_{1}}, f_{1} . \psi \in \mathcal{H}_{\mathcal{A}}^{\theta_{2}}$. Since

$$
\left(f_{1} . \psi\right)(z k, g)=f_{1}(z k, g) \psi(z k, g)=\theta_{1}(k) f_{1}(z k, g) \psi(z, g) \text {, }
$$


we demand that $\theta_{2}(k) f_{1}(z, g)=\theta_{1}(k) f_{1}(z k, g)$, or

$$
f_{1}(z k, g)=\left(\theta_{1}^{-1} \theta_{2}\right)(k) f_{1}(z, g),
$$

and $\theta_{1}^{-1} \theta_{2}$ is known directly from the structure of $\mathcal{S}$. (When $X$ is the hyperbolic plane it is the complex character describing the action of $K$ on the complex tangent space to $X$ at $u$.) A short calculation shows that

$$
\left(\left[f_{j}, \alpha\right] \cdot \psi\right)(z, g)=\int_{G}\left(f_{j}(z, g)-f_{j}\left(x^{-1} z, x^{-1} g\right)\right) \frac{\alpha(z K, x) \psi\left(x^{-1} z, x^{-1} g\right)}{\sigma\left(x, x^{-1} g\right)} d x .
$$

We observe in the next subsection that this module is 2-summable at least for kernels which decay sufficiently rapidly. Assuming this fact then it follows that $\left(\left(\omega *\left[f_{j}, \alpha\right] *\right.\right.$ $\left.\left.\left[f_{k}, \beta\right]\right) . \psi\right)(z, g)$ is given by

$$
\begin{array}{r}
\int_{G \times G \times G}\left(f_{j}\left(x^{-1} z, x^{-1} g\right)-f_{j}\left(y^{-1} z, y^{-1} g\right)\right)\left(f_{k}\left(y^{-1} z, y^{-1} g\right)-f_{k}\left(u^{-1} z, u^{-1} g\right)\right) \\
\frac{\omega(z K, x) \alpha\left(x^{-1} z K, x^{-1} y\right) \beta\left(y^{-1} z K, y^{-1} u\right)}{\sigma\left(x, x^{-1} g\right) \sigma\left(x^{-1} y, y^{-1} g\right) \sigma\left(y^{-1} u, u^{-1} g\right)} \psi\left(u^{-1} z, u^{-1} g\right) d x d y d u .
\end{array}
$$

From this (and using tr to denote the usual trace on operators on our module) we can calculate the cyclic cocycle on $\mathcal{A}$ as

$$
\tau_{c}(\omega, \alpha, \beta)=\operatorname{tr}[\epsilon \omega *[F, \alpha] *[F, \beta]]=\operatorname{tr}\left[\omega *\left(\left[f_{1}, \alpha\right] *\left[f_{2}, \beta\right]-\left[f_{2}, \alpha\right] *\left[f_{1}, \beta\right]\right)\right],
$$

which can be expressed as

$$
\int_{G / K \times G \times G} \Phi(z K, x, y) \frac{\omega(z K, x) \alpha\left(x^{-1} z K, x^{-1} y\right) \beta\left(y^{-1} z K, y^{-1}\right)}{\sigma\left(x, x^{-1} y\right) \sigma\left(y, y^{-1}\right)} d z K d x d y
$$

where

$$
\begin{aligned}
\Phi(z K, x, y) & =\int_{G}\left(f_{1}\left(x^{-1} z, x^{-1} g\right)-f_{1}\left(y^{-1} z, y^{-1} g\right)\right)\left(f_{2}\left(y^{-1} z, y^{-1} g\right)-f_{2}(z, g)\right) \\
& -\left(f_{2}\left(x^{-1} z, x^{-1} g\right)-f_{2}\left(y^{-1} z, y^{-1} g\right)\right)\left(f_{1}\left(y^{-1} z, y^{-1} g\right)-f_{1}(z, g)\right) d g .
\end{aligned}
$$

(Using the equivariance of $f_{j}$ it is easy to check that this depends on $z$ only through $z K$.) Simplifying and using $f_{1} f_{2}=1$, the integrand reduces to

$$
\begin{aligned}
& \left(f_{1}(z, g) f_{2}\left(x^{-1} z, x^{-1} g\right)+f_{1}\left(x^{-1} z, x^{-1} g\right) f_{2}\left(y^{-1} z, y^{-1} g\right)+f_{1}\left(y^{-1} z, y^{-1} g\right) f_{2}(z, g)\right) \\
- & \left(f_{2}(z, g) f_{1}\left(x^{-1} z, x^{-1} g\right)+f_{2}\left(x^{-1} z, x^{-1} g\right) f_{1}\left(y^{-1} z, y^{-1} g\right)+f_{2}\left(y^{-1} z, y^{-1} g\right) f_{1}(z, g)\right) .
\end{aligned}
$$

This can also be written more compactly as

or as

$$
\left|\begin{array}{ccc}
1 & 1 & 1 \\
f_{1}(z, g) & f_{1}\left(x^{-1} z, x^{-1} g\right) & f_{1}\left(y^{-1} z, y^{-1} g\right) \\
f_{2}(z, g) & f_{2}\left(x^{-1} z, x^{-1} g\right) & f_{2}\left(y^{-1} z, y^{-1} g\right)
\end{array}\right|
$$

$\left(1-f_{1}\left(x^{-1} z, x^{-1} g\right) f_{2}(z, g)\right)\left(1-f_{1}\left(y^{-1} z, y^{-1} g\right) f_{2}\left(x^{-1} z, x^{-1} g\right)\right)\left(1-f_{1}(z, g) f_{2}\left(y^{-1} z, y^{-1} g\right)\right)$, which also arises naturally from an alternative expression for the cocycle.

Suppose that $\varphi$ is a $U(1)$ valued function on the group, which satisfies $\varphi(k g h)=$ $\chi_{1}(k) \varphi(g) \chi_{2}(h)$ for $k$ and $h$ in $K$ and some $\sigma$-characters $\chi_{1}$ and $\chi_{2}$ of $K$. If $\chi_{1}^{-1} \chi_{2}=\theta_{1}^{-1} \theta_{2}$ 
we may take $f_{1}(z, g)=\varphi\left(z^{-1} g z\right)$ to obtain a function satisfying our earlier consistency condition.

In the case of $G=\mathbf{S U}(1,1)$ and $K$ the diagonal subgroup, we may take the function $\varphi$ used by Connes [Co2], which is essentially the Mishchenko element. With the group elements all conjugated by $z$ it now follows as in $[\mathrm{Co}$ that $\Phi(z K, x, y) / 4 \pi i$ is the area of the hyperbolic geodesic triangle with vertices $u, z^{-1} y z . u$ and $z^{-1} x z . u$. Acting with $z$ and recalling that, since $u$ is stabilised by $K, z \cdot u$ can be identified with $s=z K, \Phi(z K, x, y) / 4 \pi i$ is also the area of the geodesic triangle with vertices $s, y . s$ and $x . s$.

In the next subsection we will see that the module is 2-summable for suitably decaying kernels. Since $f_{j}\left(\gamma^{-1} z, \gamma^{-1} g \gamma\right)=\varphi\left(z^{-1} \gamma \gamma^{-1} g \gamma \gamma^{-1} z\right)=f_{j}(z, g), F$ preserves the $\Gamma$-invariant subspace, so that there is a similar expression for a cyclic cocycle $\tau_{c, \Gamma}$ in that case, except that $s$ is integrated only over the $\Gamma$ orbits in $G / K$. More precisely, using $\operatorname{tr}_{\Gamma}$ to denote this restricted range of integration, one has:

Theorem 3. There is a 2-summable Fredholm module $\left(F, \mathcal{H}_{\tau}^{\chi} \otimes \mathcal{S}\right)$ over a dense subalgebra $\mathcal{A}_{0}^{\Gamma}$ of $\mathcal{A}^{\Gamma}$, stable under the holomorphic functional calculus, whose Chern character is given by the area cocycle on $\mathbb{H}$. That is, in the notation above, one has

$$
\tau_{c, \Gamma}(\omega, \alpha, \beta)=-\operatorname{tr}_{\Gamma}[\epsilon \omega *[F, \alpha] *[F, \beta]]=-\operatorname{tr}_{\Gamma}\left[\omega *\left(\left[f_{1}, \alpha\right] *\left[f_{2}, \beta\right]-\left[f_{2}, \alpha\right] *\left[f_{1}, \beta\right]\right)\right]
$$

which can be expressed as

$$
-\int_{\Gamma \backslash G / K \times G \times G} \Phi(\Gamma z K, x, y) \frac{\omega(z K, x) \alpha\left(x^{-1} z K, x^{-1} y\right) \beta\left(y^{-1} z K, y^{-1}\right)}{\sigma\left(x, x^{-1} y\right) \sigma\left(y, y^{-1}\right)} d z K d x d y,
$$

where $\Phi$ is given as above. Therefore by the index pairing in [Co2], one has

$$
\operatorname{index}(P F P)=\left\langle\left[\tau_{c, \Gamma}\right],[P]\right\rangle,
$$

where $P$ denotes a projection in $\mathcal{A}_{0}^{\Gamma}$ and index $(P F P)$ denotes the index of the Fredholm operator PFP acting on the Hilbert space $P \mathcal{H}_{\tau}^{\chi} \otimes \mathcal{S}$.

We will prove Theorem 3 in the next subsection. The version of Theorem 3 which applies to $\mathcal{B}^{\Gamma}$ is as follows:

Theorem 4. There is a dense subalgebra $\mathcal{B}_{0}^{\Gamma}$ of $\mathcal{B}^{\Gamma}$ stable under the holomorphic functional calculus and a 2-summable Fredholm module $\left(F, \mathcal{H}_{\tau}^{\chi} \otimes \mathcal{S}\right)$ for $\mathcal{B}_{0}^{\Gamma}$ with corresponding cyclic 2-cocycle

$$
\tau_{c, \Gamma}\left(T_{\omega}, T_{\alpha}, T_{\beta}\right)=-\int_{X_{\Gamma} \times X \times X} \Phi(z, x, y) \varpi(z, x, y) T_{\omega}(z, x) T_{\alpha}(x, y) T_{\beta}(y, z) d z d x d y .
$$

The character of this Fredholm module, for $P$ a projection in $\mathcal{B}_{0}^{\Gamma}$, is given in the notation of Corollary 12 of Section 10, by

$$
\text { index }(P F P)=2(g-1)\left(\operatorname{rank} \mathcal{E}^{0}-\operatorname{rank} \mathcal{E}^{1}\right) \in \mathbb{Z},
$$

where index $(P F P)$ again denotes the index of the Fredholm operator PFP acting on the Hilbert space $P \mathcal{H}_{\tau}^{\chi} \otimes \mathcal{S}$. 
This theorem can be interpreted as an index theorem equating an analytic index with a topological index. Theorem 4 may be used to obtain the following result:

Corollary 6. Let $P$ be a projection into a gap in the spectrum of the Hamiltonian $H_{\eta, V}$. Then $P$ lies in a 2-summable dense subalgebra $\mathcal{B}_{0}^{\Gamma}$ of $\mathcal{B}^{\Gamma}$ so that in the notation of Corollary 12 of Section 10, one has

$$
\begin{aligned}
\operatorname{index}(P F P) & =\left\langle\tau_{c, \Gamma},[P]\right\rangle \\
& =2(g-1)\left(\operatorname{rank} \mathcal{E}^{0}-\operatorname{rank} \mathcal{E}^{1}\right) \in \mathbb{Z} .
\end{aligned}
$$

The statements referring to Section 10 will be clear after we establish there the hyperbolic analogues of Xia's results Xia]. The proof of the claim that the spectral projections (corresponding to gaps in the spectrum) of the Hamiltonian lie in $\mathcal{B}_{0}^{\Gamma}$ and the proof of Theorem 3 are contained in the next subsection.

7.1. Proof of summability of the Fredholm module. Here we discuss the technicalities needed for the proof of theorem 3 and of Corollary 6 . It is easy to calculate from the formulae in the previous section that 2-summability requires finiteness of the expression $\operatorname{tr}_{\Gamma}\left(\left[f_{1}, \alpha\right]^{*}\left[f_{2}, \alpha\right]\right)$, which equals

$$
\int_{X_{\Gamma} \times G \times G}\left[f_{1}(z, g)-f_{1}\left(x^{-1} z, x^{-1} g\right)\right]\left[f_{2}(z, g)-f_{2}\left(x^{-1} z, x^{-1} g\right)\right]\left|\alpha\left(x^{-1} z, x^{-1}\right)\right|^{2} d z d x d g,
$$

where $z \in X_{\Gamma}$ is a fundamental domain in $\mathbb{H}$ for the $\Gamma$ action. Letting $p: G / K \rightarrow G$ be a cross section and using the formulae for $f_{1}$ and $f_{2}$ this reduces to

$$
\int_{X_{\Gamma} \times G \times G}\left|\varphi\left(p(z)^{-1} g z\right)-\varphi\left(p(z)^{-1} g x^{-1} z\right)\right|^{2}\left|\alpha\left(x^{-1} z, x^{-1}\right)\right|^{2} d z d x d g .
$$

We are more interested in the algebra of twisted kernels as in Theorem 4, so we will present the argument for them noting that the relation for $\alpha$ in terms of $T_{\alpha}$ as given in the proof of Lemma 7 gives, by the the unitarity of $\tau$ and $\varphi$,

$$
\left|\alpha\left(x^{-1} z, x^{-1}\right)\right|^{2}=\left|T_{\alpha}\left(x^{-1} z, z\right)\right|^{2} .
$$

Thus the summability result for $\mathcal{B}^{\Gamma}$ implies that for $\mathcal{A}^{\Gamma}$.

Making this substitution we then get for our integral

$$
\int_{X_{\Gamma} \times G \times G}\left|\varphi\left(p(z)^{-1} g z\right)-\varphi\left(p(z)^{-1} g x^{-1} z\right)\right|^{2}\left|T_{\alpha}\left(x^{-1} z, z\right)\right|^{2} d z d x d g .
$$

Finally we note that setting $p(z)^{-1} g=v^{-1}$ and $x^{-1} z=w$ it is clear that $\mid \varphi\left(v^{-1} z\right)-$ $\varphi\left(v^{-1} w\right) \mid$ depends only on the cosets $v K$ and $w K$, allowing us to reduce the integral to

$$
\int_{X_{\Gamma} \times X \times X}\left|\varphi\left(v^{-1} z\right)-\varphi\left(v^{-1} w\right)\right|^{2}\left|T_{\alpha}(w, z)\right|^{2} d z d w K d v K .
$$


Only the first factor depends on $v$. Write $v=\gamma v_{0}$ for $\gamma \in \Gamma$ and $v_{0} \in X_{\Gamma}$. Then we obtain for our integral after a change of variables:

$$
\sum_{\gamma \in \Gamma} \int_{\gamma \cdot X_{\Gamma} \times X \times X_{\Gamma}}\left|\varphi\left(v_{0}^{-1} z\right)-\varphi\left(v_{0}^{-1} w\right)\right|^{2}\left|T_{\alpha}(\gamma w, \gamma z)\right|^{2} d z d w K d v_{0} K
$$

so that by the $\gamma$ invariance of the kernel $T_{\alpha}$ one obtains

$$
\int_{X \times X \times X_{\Gamma}}\left|\varphi\left(v_{0}^{-1} z\right)-\varphi\left(v_{0}^{-1} w\right)\right|^{2}\left|T_{\alpha}(w, z)\right|^{2} d z d w K d v_{0} K
$$

By a further change of variable we obtain

$$
\int_{X \times X \times X_{\Gamma}}|\varphi(z)-\varphi(w)|^{2}\left|T_{\alpha}\left(v_{0}^{-1} w, v_{0}^{-1} z\right)\right|^{2} d z d w K d v_{0} K
$$

Notice that, by lemma $6, T_{\alpha}\left(v_{0}^{-1} w, v_{0}^{-1} z\right)$ is the integral kernel for the operator obtained by conjugating by $U\left(v_{0}\right)$. It follows therefore that finiteness of the triple integral is guaranteed by the convergence of

$$
\int_{X \times X}|\varphi(z)-\varphi(w)|^{2}\left|T_{\alpha}(w, z)\right|^{2} d z d w K
$$

To avoid repetition let us first focus on the case of greatest interest where we consider the integral kernels of a spectral projection $P$ of the Hamiltonian $H+V$ corresponding to a gap in the spectrum. As it is obtained from the Hamiltonian using the smooth functional calculus from a function of compact support we can obtain a growth estimate on the integral kernel (see below) which will ensure convergence. To lighten the notation we let $z, w \in X$ and $k(z, w)$ denote the integral kernel as a function on $X \times X$. Taking $\chi_{1}=1$ so that $\psi(g k)=\varphi(g)$ is well defined, the discussion of the previous paragraph leads us to consider whether

$$
\iint|(\psi(z)-\psi(w)) k(z, w)|^{2} d z d w
$$

is finite. Let $X_{0}$ be a fundamental domain for the diagonal action of $\Gamma$ on $X \times X$. Then the previous integral, for $\Gamma$ invariant kernels, is given by

$$
\sum_{\gamma \in \Gamma} \iint_{X_{0}}|(\psi(\gamma . z)-\psi(\gamma \cdot w)) k(z, w)|^{2} d z d w .
$$

By an argument due to Connes $\mathrm{CO}$, we have the estimate

$$
|\psi(\gamma . z)-\psi(\gamma . w)|^{2} \leq C \exp \left(-2 d(u, \gamma . z)+C_{1} d(\gamma . z, \gamma \cdot w)\right) .
$$

(Here $u$ is the base point in $X, d$ denotes the hyperbolic metric and $C, C_{1}$ are constants.)

We claim that, in addition, the following estimate holds:

$$
|k(z, w)|^{2} \leq C_{2} \exp \left(-C_{3} d(z, w)^{2}\right),
$$

where $C_{2}, C_{3}$ are constants. This fact goes back to CGT although in the form we need it here, for the Hamiltonians $H+V$ of Section 4, it can be deduced from $B$ BrSu. This is because $\mathrm{BrSu}$ prove $(* *)$ when $k$ is the kernel of the heat operator $e^{-(H+V)}$. Now by the argument of Corollary 3 there is a smooth function of compact support $g$ such that 
$g\left(e^{-(H+V)}\right)$ is the spectral projection $P$. To prove that the kernel of $g\left(e^{-(H+V)}\right)$ satisfies $(* *)$ it suffices to observe that we can approximate $g$ uniformly by polynomials without constant term so that the kernel of $g\left(e^{-(H+V)}\right)$ has the same off-diagonal decay estimate as the kernel of $e^{-(H+V)}$, namely $(* *)$.

Hence the integral in $\left(^{*}\right)$ above is smaller than

$$
\sum_{\gamma \in \Gamma} \iint_{X_{0}} C_{4} \exp \left(-C_{3} d(z, w)^{2}+C_{1} d(z, w)-2 d(u, \gamma . z)\right) \quad(* * *)
$$

for suitable constants $C_{j}, j=1,2,3$. As the area in hyperbolic space grows like $\exp (d(u, z))$, convergence of the infinite sum in $(* * *)$ is handled by the convergence of the Poincare series $\sum_{\gamma \in \Gamma} \exp (-2 d(u, \gamma . z))$. The convergence of the integral in $(* * *)$, over the fundamental domain, is handled by the exponential factor involving the square of the hyperbolic distance and noting that the integration in the diagonal direction in $X_{0}$ is over a finite range. (It is also possible to prove 2-summability in the case when $\Gamma$ is trivial by exploiting the fact that in that case one may use kernels with restrictions on their support.)

Since operators with kernels which have support in a band around the diagonal are dense in the algebras $\mathcal{A}^{\Gamma}$ and $\mathcal{B}^{\Gamma}$ so too is the set of operators with kernels satisfying (**). Now the finiteness of $(*)$ is equivalent to asserting that $\left[F, T_{\alpha}\right]$ is Hilbert-Schmidt.

Definition. We denote by $\mathcal{B}_{0}^{\Gamma}$ the subalgebra consisting of operators $A \in \mathcal{B}^{\Gamma}$, with $[F, A]$ a Hilbert-Schmidt operator.

The argument of the previous paragraph shows that $\mathcal{B}_{0}^{\Gamma}$ is dense. Now by $\mathrm{Co} \mathcal{B}_{0}^{\Gamma}$ is stable under the holomorphic functional calculus. A similar remark handles the existence of the analogous dense subalgebra $\mathcal{A}_{0}^{\Gamma}$ of $\mathcal{A}^{\Gamma}$. This completes the proof of Theorem 3 and the claim concerning the spectral projections of the Hamiltonian as we promised.

7.2. The hyperbolic Connes-Kubo formula. Our aim in this subsection is to give a geometric interpretation to the cocycles defined in lemma 12 and to prove that a suitable linear combination of them is cohomologous to the cocycle $\tau_{c, \Gamma}$ arising from the Fredholm module $\left(F, \mathcal{H}_{\tau}^{\chi} \otimes \mathcal{S}\right)$.

To do this we begin by introducing, for operators $A_{0}, A_{1}, A_{2}$ in $\mathcal{B}^{\Gamma}$ whose kernels $k_{0}, k_{1}, k_{2}$ are exponentially decaying (cf equation (**) of the previous subsection) the cyclic cocycle $c_{K}$ defined by

$$
\begin{aligned}
c_{K}\left(A_{0}, A_{1}, A_{2}\right) & =\sum_{j=1}^{g} c_{j, j+g}\left(A_{0}, A_{1}, A_{2}\right) \\
& =\sum_{j=1}^{g} \int_{X_{\Gamma} \times X \times X} \varpi(z, x, y) \Psi_{j}(z, x, y) k_{0}(z, x) k_{1}(x, y) k_{2}(y, z) d z d x d y,
\end{aligned}
$$

where

$\Psi_{j}(z, x, y)=\left(\Omega_{j}(x)-\Omega_{j}(y)\right)\left(\Omega_{j+g}(y)-\Omega_{j+g}(z)\right)-\left(\Omega_{j+g}(x)-\Omega_{j+g}(y)\right)\left(\Omega_{j}(y)-\Omega_{j}(z)\right)$. 
We claim that $\sum_{j=1}^{g} \Psi_{j}(z, x, y)$ is proportional to the 'symplectic area' of a triangle in $\mathbb{R}^{2 g}$ with vertices $\Xi(x), \Xi(y), \Xi(z)$.

To prove this it suffices to assume that the origin is one of the vertices of the triangle, so suppose $z$ is the base point in $\mathbb{H}$. Then we need to consider the expression

$$
\sum_{j=1}^{g} \Psi_{j}(z, x, y)=\sum_{j=1}^{g}\left(\Omega_{j}(x) \Omega_{j+g}(y)-\Omega_{j+g}(x) \Omega_{j}(y)\right) .
$$

Let $s$ denote the symplectic form on $\mathbb{R}^{2 g}$ given by:

$$
s(u, v)=\sum_{j=1}^{g}\left(u_{j} v_{j+g}-u_{j+g} v_{j}\right)
$$

The so-called 'symplectic area' of a triangle with vertices $0, \Xi(x), \Xi(y)$ may be seen to be $s(\Xi(x), \Xi(y))$. To appreciate this, however, we need to utilise an argument from GH (pp $333-336$ ). In terms of the standard basis of $\mathbb{R}^{2 g}$ (given in this case by vertices in the integer period lattice arising from our choice of basis of harmonic one forms) and corresponding coordinates $u_{1}, u_{2}, \ldots u_{2 g}$ the form $s$ is the two form on $\mathbb{R}^{2 g}$ given by

$$
\omega_{J}=\sum_{j=1}^{g} d u_{j} \wedge d u_{j+g}
$$

Now the 'symplectic area' of a triangle in $\mathbb{R}^{2 g}$ with vertices $0, \Xi(x), \Xi(y)$ is given by integrating $\omega_{J}$ over the triangle and a brief calculation reveals that this yields $s(\Xi(x), \Xi(y)) / 2$, proving our claim.

The previous argument establishes the following result.

Proposition 2. The higher genus analogue of the Kubo formula is given by the cyclic cocycle $\tau_{K}$ on $\mathcal{B}^{\Gamma}$ defined by

$$
\begin{aligned}
\tau_{K}\left(A_{0}, A_{1}, A_{2}\right) & =\sum_{j=1}^{g} c_{j, j+g}\left(A_{0}, A_{1}, A_{2}\right) \\
& =\sum_{j=1}^{g} \int_{X_{\Gamma} \times X \times X} \Psi_{j}(z, x, y) \varpi(z, x, y) k_{0}(z, x) k_{1}(x, y) k_{2}(y, z) d z d x d y .
\end{aligned}
$$

Here the $k_{j}$ are the kernels of the $A_{j}, j=0,1,2$ (three exponentially decaying elements of $\left.\mathcal{B}^{\Gamma}\right)$ and $\sum_{j=1}^{g} \Psi_{j}(z, x, y)$ is proportional to the 'symplectic area' of the Euclidean triangle $\Delta_{E}$ in $\mathbb{R}^{2 g}$ with vertices $\Xi(x), \Xi(y), \Xi(z)$.

To compare the cocycle $\tau_{K}$ with the cocycle $\tau_{c, \Gamma}$ arising from our Fredholm module we note that the pull back form $\Xi^{*}\left(d u_{j}\right)$ is dual to the homology cycle $A_{j}$ for $j=1, \ldots, g$ and dual to $B_{j-g}$ for $j=g+1, \ldots, 2 g$ (cf [GH]). Thus $\Xi^{*}\left(d u_{j}\right)$ differs from $a_{j}$ by an exact one form. Hence $\Xi^{*}\left(\omega_{J}\right)$ differs from $\sum_{j=1}^{g} a_{j} \wedge a_{j+g}$ by an exact two form. But each term 
$a_{j} \wedge a_{j+g}$ is harmonic and hence proportional to the two form $\omega_{\mathbb{H}}$ on $\mathbb{H}$. So we have for some constant $\kappa$, and geodesic triangle $\Delta \subset \mathbb{H}$,

$$
\int_{\Delta} \omega_{\mathbb{H}}=\kappa \int_{\Delta} \Xi^{*}\left(\omega_{J}\right)=\kappa \int_{\Xi(\Delta)} \omega_{J}
$$

Actually a calculation reveals that one can do a little better than this and proves that

$$
\kappa \Xi^{*}\left(\omega_{J}\right)=\omega_{\mathbb{H}} .
$$

Now $\Xi$ cannot map geodesic triangles to Euclidean triangles in $\mathbb{R}^{2 g}$ as $\Xi(\Delta)$ is a compact subset of a non-flat embedded two dimensional surface in $\mathbb{R}^{2 g}$. Moreover as $\Psi_{j}(z, x, y)=0$ whenever the images of $z, x, y$ under $\Xi$ lie in a Lagrangian subspace (with respect to the symplectic form $s$ ) of $\mathbb{R}^{2 g}, \tau_{K}$ and $\tau_{c, \Gamma}$ are not obviously proportional.

After suitable normalisation we will, however, prove they are cohomologous. First renormalise $\omega_{J}$ so that $\Xi^{*}\left(\omega_{J}\right)=\omega_{\mathbb{H}}$ and then normalise $\sum_{j=1}^{g} \Psi_{j}(z, x, y)$ so that it equals $-4 \pi i \int_{\Delta_{E}} \omega_{J}$. Next we write $\omega_{J}=d \theta$. Considering the difference $\tau_{K}-\tau_{c, \Gamma}$ one sees that the key is to understand

$$
\int_{\Xi(\Delta)} \omega_{J}-\int_{\Delta_{E}} \omega_{J}=\int_{\partial \Xi(\Delta)} \theta-\int_{\partial \Delta_{E}} \theta
$$

Now this difference of integrals around the boundary can be written as the sum of three terms corresponding to splitting the boundaries $\partial \Xi(\Delta)$ and $\partial \Delta_{E}$ into three arc segments each. We introduce some notation for this, writing

$$
\partial \Xi(\Delta)=\Xi(\ell(x, y)) \cup \Xi(\ell(y, z)) \cup \Xi(\ell(z, x)),
$$

where $\ell(x, y)$ is the geodesic in $\mathbb{H}$ joining $x$ and $y$ (with the obvious similar definition of the other terms). We also write

$$
\partial \Delta_{E}=m(x, y) \cup m(y, z) \cup m(z, x),
$$

where $m(x, y)$ is the straight line joining $\Xi(x)$ and $\Xi(y)$ (and again the obvious definition of the other terms). Then we have

$$
\int_{\partial \Xi(\Delta)} \theta-\int_{\partial \Delta_{E}} \theta=h(x, y)+h(y, z)+h(z, x)
$$

where $h(x, y)=\int_{\Xi(\ell(x, y)} \theta-\int_{m(x, y)} \theta$ with similar definitions for $h(y, z)$ and $h(z, x)$.

Notice that we can write $h(x, y)=\int_{D_{x y}} \omega_{J}$ where $D_{x y}$ is a disc with boundary $m(x, y) \cup$ $\ell(x, y)$. From this it is easy to see that $h(\gamma x, \gamma y)=h(x, y)$ for $\gamma \in \Gamma$. Introduce the bilinear functional $\tau_{1}$ on $\mathcal{B}^{\Gamma}$ given by

$$
\tau_{1}\left(A_{0}, A_{1}\right)=-4 \pi i \int_{X_{\Gamma} \times X} h(x, y) k_{0}(x, y) k_{1}(y, x) d x d y=-4 \pi i \operatorname{tr}_{\mathcal{B}^{\Gamma}}\left(A_{h} A_{1}\right),
$$

where, if $A_{0}$ has kernel $k_{0}(x, y), A_{h}$ is the operator with kernel $h(x, y) k_{0}(x, y)$. Of course this definition begs the question of whether the trace is finite. 
In order to prove that $\tau_{1}$ is densely defined we start with some preliminary observations. By $\mathrm{M}+\mathrm{R}+\mathrm{W}$ and Ren2 there is an isomorphism

$$
\Phi_{F}: \mathcal{B}^{\Gamma} \cong C_{r}^{*}\left(\Gamma_{g}, \sigma\right) \otimes \mathcal{K}\left(L^{2}(F)\right) .
$$

Here $F$ denotes a fundamental domain for the action of $\Gamma_{g}$ on $\mathbb{H}$. (Note that by the PackerRaeburn stabilization theorem, one has $\left.C_{r}^{*}\left(\Gamma_{g}, \sigma\right) \otimes \mathcal{K} \cong \mathcal{K} \rtimes_{r} \Gamma_{g}.\right)$ Now any element $x$ in $C_{r}^{*}\left(\Gamma_{g}, \sigma\right) \otimes \mathcal{K}$ can be written as a matrix $\left(x_{i j}\right)$, where $x_{i j} \in C_{r}^{*}\left(\Gamma_{g}, \sigma\right)$. So we can define

$$
N_{k}(x)=\left(\sum_{i, j} \nu\left(x_{i j}\right)^{2}\right)^{\frac{1}{2}}
$$

where

$$
\nu\left(x_{i j}\right)=\left(\sum_{h \in \Gamma_{g}}\left(1+\ell(h)^{2 k}\right)|x(h)|^{2}\right)^{\frac{1}{2}}
$$

and $\ell$ denotes the word length function on the group $\Gamma_{g}$. By a mild modification of the argument given in Co2], III.5. $\gamma$, one can prove that there is a subalgebra $\mathcal{B}_{\infty}^{\Gamma}$ of $\mathcal{B}^{\Gamma}$ which contains $\mathbb{C}\left(\Gamma_{g}, \sigma\right) \otimes \mathcal{R}$, where $\mathcal{R}$ denotes the algebra of smoothing operators on $F$, is stable under the holomorphic functional calculus, and is such that $N_{k}(x)<\infty$ for all $x \in \mathcal{B}_{\infty}^{\Gamma}$ and $k \in \mathbb{N}$. Then one shows as in Co2 that the trace $\tau \otimes \operatorname{Tr}$ on $\mathbb{C}\left(\Gamma_{g}, \sigma\right) \otimes \mathcal{R}$, is continuous for the norm $N_{k}$, for $k$ sufficiently large, and thus extends by continuity to $\mathcal{B}_{\infty}^{\Gamma}$. Note that elements in $\mathcal{B}_{\infty}^{\Gamma}$ have Schwartz kernels which have rapid decay away from the diagonal. An alternate equivalent construction of $\mathcal{B}_{\infty}^{\Gamma}$ would be to use the algebra $A_{g, \sigma}$ as in Section 10 , and the results of [Ji].

Summarizing this, we have

Proposition 3. The algebra $\mathcal{B}_{\infty}^{\Gamma}$ is dense in $\mathcal{B}^{\Gamma}$, is closed under the holomorphic functional calculus and is contained in the ideal $\mathcal{I}$ of $\mathcal{B}^{\Gamma}$ consisting of operators with finite trace.

Now $\tau_{K}$ is defined on $\mathcal{B}_{\infty}^{\Gamma}$ while $\tau_{c, \Gamma}$ is defined on $\mathcal{B}_{0}^{\Gamma}$ as we noted earlier. Both of these algebras contain the operators whose Schwartz kernels are supported in a band around the diagonal. Thus the subalgebra $\mathcal{B}_{\infty}^{\Gamma} \cap \mathcal{B}_{0}^{\Gamma}$ is dense and stable under the holomorphic functional calculus. If $b$ denotes the Hochschild coboundary map then a straightforward calculation reveals that $b \tau_{1}=\tau_{K}-\tau_{c, \Gamma}$. The Lipschitz property of the Jacobi map means that $h(x, y)$ grows at worst like the square of the hyperbolic distance from $x$ to $y$ so that if $A_{0} \in \mathcal{B}_{\infty}^{\Gamma}$ then so too does $A_{h}$. Hence we have $\tau_{1}$ defined on $\mathcal{B}_{\infty}^{\Gamma} \cap \mathcal{B}_{0}^{\Gamma}$ proving the following theorem.

Theorem 5. The Kubo cocycle $\tau_{K}$ and the Chern character cocycle $\tau_{c, \Gamma}$ arising as the Chern class of the Fredholm module $\left(F, \mathcal{H}_{\tau}^{\chi} \otimes \mathcal{S}\right)$, are cohomologous as cyclic cocycles on $\mathcal{B}_{\infty}^{\Gamma} \cap \mathcal{B}_{0}^{\Gamma}$.

This theorem replaces the Connes-Kubo formula in genus one. The latter formula states that the two cocycles of the theorem are equal. We see that the situation is more complex for genus $g$ but from the viewpoint of $K$-theory as described in the next section this theorem is enough to give integrality of the Hall conductivity defined either from the character of our Fredholm module or from the hyperbolic Kubo formula. 


\section{8. $K$-THEORY ASPECTS}

In this section, we compute the $K$-groups of the twisted group $C^{*}$-algebras which are relevant to the quantum Hall effect on the hyperbolic plane as a special case of more general theorems about the $K$-groups of the twisted group $C^{*}$-algebras of groups $\Gamma$ which are uniform lattices in $K$-amenable Lie groups.

We recall that any solvable Lie group, and in fact any amenable Lie group is $K$ amenable. However, it has been proved by Kasparov [Kas1] in the case of the nonamenable groups $\mathbf{S O}_{0}(n, 1)$ and by Julg-Kasparov JuKas in the case of $\mathbf{S U}(n, 1)$ that these are $K$-amenable Lie groups. Cuntz $\mathbb{C u}$ has shown that the class of $K$-amenable groups is closed under the operations of taking subgroups, under free products and under direct products. Our method uses the $K$-amenability results of Kasparov [Kas] and the Packer-Raeburn stabilization theorem [PR]. In [PR1], one can find an example where the twisted $K$-theory $K^{*}\left(\Gamma \backslash G / K, \delta\left(B_{\sigma}\right)\right)$ is not isomorphic to $K^{*}(\Gamma \backslash G / K)$, even when $G$ is the $K$-amenable solvable group $\mathbb{R}^{n} \rtimes \mathbb{R}, K=\{e\}, \Gamma=\mathbb{Z}^{n} \rtimes \mathbb{Z}$ and for some multiplier $\sigma$ on $\Gamma$ with non-trivial Dixmier-Douady invariant $\delta\left(B_{\sigma}\right)=\delta(\sigma) \neq 0$. However, $K^{*}\left(\Gamma \backslash G / K, \delta\left(B_{\sigma}\right)\right)$ is isomorphic to $K^{*}(\Gamma \backslash G / K)$ whenever the Dixmier-Douady invariant $\delta\left(B_{\sigma}\right)=\delta(\sigma)=0$ is trivial. We identify the Dixmier-Douady invariant $\delta\left(B_{\sigma}\right)$ with the image of $\sigma$ under the connecting homomorphism $\delta: H^{2}(\Gamma, U(1)) \rightarrow H^{3}(\Gamma, \mathbb{Z})$ of the change of coefficients exact sequence in cohomology, corresponding to the short exact sequence of coefficient groups

$$
1 \rightarrow \mathbb{Z} \stackrel{i}{\rightarrow} \mathbb{R} \stackrel{e^{2 \pi \sqrt{-1}}}{\longrightarrow} U(1) \rightarrow 1
$$

This enables us to prove vanishing theorems for the Dixmier-Douady invariant whenever $\Gamma$ is a lattice in a connected Lie group $G$ such that $\operatorname{dim}(G / K) \leq 3$, where $K$ is a maximal compact subgroup of $G$, and therefore we obtain in this case

$$
K_{*}\left(C^{*}(\Gamma, \sigma)\right) \cong K^{*+\operatorname{dim}(G / K)}(\Gamma \backslash G / K)
$$

where $\sigma$ is any multiplier on $\Gamma$. This is the case for the Riemann surfaces which are the object of our study in this paper.

We begin by reviewing the concept of $K$-amenable groups. Let $G$ be a connected Lie group and $K$ be a maximal compact subgroup. For our purposes, we will assume that $\operatorname{dim}(G / K)$ is even, and that it has a $G$-invariant $\operatorname{Spin}^{\mathbb{C}}$ structure. Using the Spin ${ }^{\mathbb{C}}$ structure, we can form the $G$-invariant Dirac operator $\not \partial$ on $G / K$. It is a first order, selfadjoint, elliptic differential operator acting on $L^{2}$ sections of the $\mathbb{Z}_{2}$ graded homogeneous bundle of spinors $S$. Consider $F=\not \partial\left(1+\not \partial^{2}\right)^{-1 / 2}$, which is a 0 th order pseudo-differential operator acting on $H=L^{2}(G / K, S)$. $C_{0}(G / K)$ acts on $H$ by multiplication operators, $f \rightarrow M_{f}$. Also $G$ acts on $C_{0}(G / K)$ and on $H$ by left translation, and $F$ is $G$-invariant. Therefore $(H, M, F)$ defines a canonical element, called the Dirac element,

$$
\alpha_{G} \in K K_{G}\left(C_{0}(G / K), \mathbb{C}\right)
$$

Theorem 6 ( Kas2]). There is a canonical element, called the Mishchenko element

$$
\beta_{G} \in K K_{G}\left(\mathbb{C}, C_{0}(G / K)\right),
$$


such that one has the following intersection products:

(1) $\alpha_{G} \otimes_{\mathbb{C}} \beta_{G}=1_{C_{0}(G / K)} \in K K_{G}\left(C_{0}(G / K), C_{0}(G / K)\right)$

(2) $\beta_{G} \otimes_{C_{0}(G / K)} \alpha_{G}=\gamma_{G} \in K K_{G}(\mathbb{C}, \mathbb{C})$ where $\gamma_{G}$ is an idempotent in $K K_{G}(\mathbb{C}, \mathbb{C})$.

The Mishchenko element $\beta_{G}$ can be described as follows. First assume that either $G$ is semisimple or that $G=\mathbb{R}^{n}$. Then the Killing form on $G$ defines a $G$-invariant Riemannian metric of non-positive sectional curvature on $G / K$. Let $\mathcal{E}=C_{0}\left(G / K, \mathcal{S}^{*}\right)$ be the space of continuous sections of the dual spin bundle $\mathcal{S}^{*}$ which vanish at infinity. Let $F$ be a bounded operator on $\mathcal{E}$ defined as

$$
F \xi(x)=c\left(V\left(x, x_{0}\right)\right) \xi(x),
$$

where $\xi \in \mathcal{E}, V\left(x, x_{0}\right) \in T_{x}(G / K)$ is the unit vector which is tangent to the unique geodesic from $x_{0} \in G / K$ to $x$ and $c\left(V\left(x, x_{0}\right)\right)$ denotes Clifford multiplication by $V\left(x, x_{0}\right)$. Then $V\left(x, x_{0}\right)$ is well defined outside a small neighbourhood of $x_{0}$ and can be extended continuously in any way to all of $G / K$. As $F$ is adjointable it lies in $\mathcal{L}(\mathcal{E})$. Also since

$$
F^{2} \xi(x)=\left\|V\left(x, x_{0}\right)\right\|^{2} \xi(x),
$$

we see that $F^{2}-1 \in \mathcal{K}(\mathcal{E})$ is a compact operator in $\mathcal{L}(\mathcal{E})$. For $g \in G$, define

$$
(g . F) \xi(x)=c\left(V\left(x, g x_{0}\right)\right) \xi(x) .
$$

Since $G / K$ has negative sectional curvature, the function on $G / K$ defined by

$$
x \rightarrow\left\|V\left(x, x_{0}\right)-V\left(x, x_{1}\right)\right\|, \quad x_{0}, x_{1} \in G / K,
$$

vanishes at infinity, and so is in $C_{0}(G / K)$. Therefore $g . F-F \in \mathcal{K}(\mathcal{E})$ and $(\mathcal{E}, F)$ defines an element $\beta_{G} \in K K_{G}\left(\mathbb{C}, C_{0}(G / K)\right)$. The Mishchenko element $\beta_{G}$ is constructed by induction in the general case.

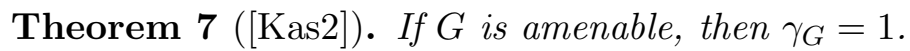

This motivates the following definition ( Kas2]).

Definition. A Lie group $G$ is said to be $K$-amenable if $\gamma_{G}=1$.

Theorem 8 ([Kas1]JuKas]). The non-amenable groups $\mathbf{S O}(n, 1)$ and $\mathbf{S U}(n, 1)$ are $K$ amenable.

Let $\Gamma \subset G$ be a lattice in $G$ and $A$ be an algebra admitting an automorphic action of $\Gamma$. Then the cross product algebra $\left[A \otimes C_{0}(G / K)\right] \rtimes \Gamma$, is Morita equivalent to the algebra of continuous sections vanishing at infinity $C_{0}(\Gamma \backslash G / K, \mathcal{E})$, where $\mathcal{E} \rightarrow \Gamma \backslash G / K$ is the flat $A$-bundle defined as the quotient

$$
\mathcal{E}=(A \times G / K) / \Gamma \rightarrow \Gamma \backslash G / K .
$$

Here we consider the diagonal action of $\Gamma$ on $A \times G / K$.

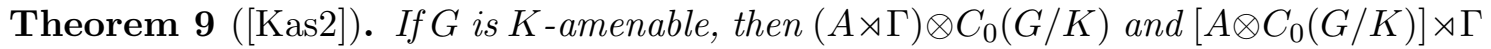
have the same K-theory. 
Combining Theorem 9 with the remarks above, one gets the following important corollary.

Corollary 7. If $G$ is $K$-amenable, then $(A \rtimes \Gamma) \otimes C_{0}(G / K)$ and $C_{0}(\Gamma \backslash G / K, \mathcal{E})$ have the same $K$-theory. Equivalently, one has for $j=0,1$,

$$
K_{j}\left(C_{0}(\Gamma \backslash G / K, \mathcal{E})\right) \cong K_{j+\operatorname{dim}(G / K)}(A \rtimes \Gamma) .
$$

We now come to the main theorem of this section, which generalizes theorems of [PR], [PR2].

Theorem 10. Suppose that $\Gamma$ is a lattice in a $K$-amenable Lie group $G$ and that $K$ is a maximal compact subgroup of $G$. Then

$$
K_{*}\left(C^{*}(\Gamma, \sigma)\right) \cong K^{*+\operatorname{dim}(G / K)}\left(\Gamma \backslash G / K, \delta\left(B_{\sigma}\right)\right),
$$

where $\sigma \in H^{2}(\Gamma, U(1))$ is any multiplier on $\Gamma, K^{*}\left(\Gamma \backslash G / K, \delta\left(B_{\sigma}\right)\right)$ is the twisted $K$ theory of a continuous trace $C^{*}$-algebra $B_{\sigma}$ with spectrum $\Gamma \backslash G / K$, and $\delta\left(B_{\sigma}\right)$ denotes the Dixmier-Douady invariant of $B_{\sigma}$.

Proof. 1. Taking the case $A=\mathbb{C}$ and the trivial action of $\Gamma$ on $\mathbb{C}$, one sees by Corollary 7 that $C^{*}(\Gamma)$ and $C_{0}(\Gamma \backslash G / K)$ have the same $K$-theory when $\gamma_{G}=1$.

2. Let $\sigma \in H^{2}(\Gamma, U(1))$, then the twisted cross product algebra $A \rtimes_{\sigma} \Gamma$ is stably equivalent to $(A \otimes \mathcal{K}) \rtimes \Gamma$ where $\mathcal{K}$ denotes compact operators. This is the PackerRaeburn stabilization trick [PR] (note that the $\Gamma$ action on $\mathcal{K}$ takes some time to describe and we refer the reader to $\left[\mathrm{PR}\right.$ for details). Using Corollary 7 again, one sees that $A \rtimes_{\sigma}$ $\Gamma \otimes C_{0}(G / K)$ and $C_{0}\left(\Gamma \backslash G / K, \mathcal{E}_{\sigma}\right)$ have the same $K$-theory, whenever $G$ is $K$-amenable, where

$$
\mathcal{E}_{\sigma}=(A \otimes \mathcal{K} \times G / K) / \Gamma \rightarrow \Gamma \backslash G / K
$$

is a flat $A \otimes \mathcal{K}$-bundle over $\Gamma \backslash G / K$ and $K$ is a maximal compact subgroup of $G$. In the particular case when $A=\mathbb{C}$, one sees that $C_{r}^{*}(\Gamma, \sigma) \otimes C_{0}(G / K)$ and $C_{0}\left(\Gamma \backslash G / K, \mathcal{E}_{\sigma}\right)$ have the same $K$-theory whenever $G$ is $K$-amenable, where

$$
\mathcal{E}_{\sigma}=(\mathcal{K} \times G / K) / \Gamma \rightarrow \Gamma \backslash G / K .
$$

But the twisted $K$-theory $K^{*}\left(\Gamma \backslash G / K, \delta\left(B_{\sigma}\right)\right)$ is by definition the $K$-theory of the continuous trace $C^{*}$-algebra $B_{\sigma}=C_{0}\left(\Gamma \backslash G / K, \mathcal{E}_{\sigma}\right)$ with spectrum $\Gamma \backslash G / K$. Then

$$
K_{*}\left(C^{*}(\Gamma, \sigma)\right) \cong K^{*+\operatorname{dim}(G / K)}\left(\Gamma \backslash G / K, \delta\left(B_{\sigma}\right)\right) .
$$

Remarks. Consider the flat case, when $G=\mathbb{R}^{2 n} \rtimes \mathbf{S O}(2 n)$ is the Euclidean group, $K=\mathbf{S O}(2 n)$, and $\Gamma \subset G$ is a Bieberbach group, that is, $\Gamma$ is a uniform lattice in $G$. One can define a generalization of "noncommutative flat manifolds" by regarding $C^{*}(\Gamma, \sigma)$ as such an object, where $\sigma$ is any group 2-cocycle on $\Gamma$, by virtue of the fact that

$$
K_{*}\left(C^{*}(\Gamma, \sigma)\right) \cong K^{*}(\Gamma \backslash G / K) .
$$


Our next main result says that for lattices in $K$-amenable Lie groups, the reduced and unreduced twisted group $C^{*}$-algebras have canonically isomorphic $K$-theories. Therefore all the results that we prove regarding the $K$-theory of these reduced twisted group $C^{*}$ algebras are also valid for the unreduced twisted group $C^{*}$-algebras.

Theorem 11. Let $\sigma \in H^{2}(\Gamma, U(1))$ be a multiplier on $\Gamma$ and $\Gamma$ be a lattice in a $K$ amenable Lie group. Then the canonical morphism $C^{*}(\Gamma, \sigma) \rightarrow C_{r}^{*}(\Gamma, \sigma)$ induces an isomorphism

$$
K_{*}\left(C^{*}(\Gamma, \sigma)\right) \cong K_{*}\left(C_{r}^{*}(\Gamma, \sigma)\right)
$$

Proof. We note that by the Packer-Raeburn trick, one has

$$
C^{*}(\Gamma, \sigma) \otimes \mathcal{K} \cong \mathcal{K} \rtimes \Gamma
$$

and

$$
C_{r}^{*}(\Gamma, \sigma) \otimes \mathcal{K} \cong \mathcal{K} \rtimes_{r} \Gamma
$$

where $\rtimes_{r}$ denotes the reduced crossed product. Since $\Gamma$ is a lattice in a $K$-amenable Lie group, the canonical morphism $\mathcal{K} \rtimes \Gamma \rightarrow \mathcal{K} \rtimes_{r} \Gamma$ induces an isomorphism

$$
K_{*}(\mathcal{K} \rtimes \Gamma) \cong K_{*}\left(\mathcal{K} \rtimes_{r} \Gamma\right)
$$

which proves the result.

We now specialize to the case when $G=\mathbf{S O}_{0}(2,1), K=\mathbf{S O}(2)$ and $\Gamma=\Gamma_{g}$ is the fundamental group of a Riemann surface of genus $g>1, \Sigma_{g}$, where $\Gamma_{g} \subset G$ and $G$ is $K$-amenable, or when $G=\mathbb{R}^{2}, K=\{e\}$ and $g=1$, with $\Gamma_{1}$ being $\mathbb{Z}^{2}$.

Corollary 8. Let $\sigma \in H^{2}\left(\Gamma_{g}, U(1)\right)$ be any multiplier on $\Gamma_{g}$. Then

1. $K_{0}\left(C_{r}^{*}\left(\Gamma_{g}, \sigma\right)\right) \cong K_{0}\left(C_{r}^{*}\left(\Gamma_{g}\right)\right) \cong K^{0}\left(\Sigma_{g}\right) \cong \mathbb{Z}^{2}$

2. $K_{1}\left(C_{r}^{*}\left(\Gamma_{g}, \sigma\right)\right) \cong K_{1}\left(C_{r}^{*}\left(\Gamma_{g}\right)\right) \cong K^{1}\left(\Sigma_{g}\right) \cong \mathbb{Z}^{2 g}$.

Proof. In dimension 2 the Chern character is an isomorphism over the integers and therefore we see that

$$
K^{0}\left(\Sigma_{g}\right) \cong H^{0}\left(\Sigma_{g}, \mathbb{Z}\right) \oplus H^{2}\left(\Sigma_{g}, \mathbb{Z}\right) \cong \mathbb{Z}^{2}
$$

and that

$$
K^{1}\left(\Sigma_{g}\right) \cong H^{1}\left(\Sigma_{g}, \mathbb{Z}\right) \cong \mathbb{Z}^{2 g}
$$

By Theorem 10 we have

$$
K_{j}\left(C_{r}^{*}\left(\Gamma_{g}\right)\right) \cong K^{j}\left(\Sigma_{g}\right) \text { for } j=0,1
$$

and

$$
K_{j}\left(C_{r}^{*}\left(\Gamma_{g}, \sigma\right)\right) \cong K_{j}\left(\Sigma_{g}, \delta\left(B_{\sigma}\right)\right), \quad j=0,1,
$$

where $B_{\sigma}=C\left(\Sigma_{g}, \mathcal{E}_{\sigma}\right)$. Finally, because $\mathcal{E}_{\sigma}$ is a locally trivial flat bundle of $C^{*}$-algebras over $\Sigma_{g}$, with fibre $\mathcal{K}$ (= compact operators), it has a Dixmier-Douady invariant $\delta\left(B_{\sigma}\right)$ which can be viewed as the obstruction to $B_{\sigma}$ being Morita equivalent to $C\left(\Sigma_{g}\right)$. But

$$
\delta\left(B_{\sigma}\right)=\delta(\sigma) \in H^{3}\left(\Sigma_{g}, \mathbb{Z}\right)=0 .
$$


Therefore $B_{\sigma}$ is Morita equivalent to $C\left(\Sigma_{g}\right)$ and we conclude that

$$
K_{j}\left(C_{r}^{*}\left(\Gamma_{g}, \sigma\right)\right) \cong K^{j}\left(\Sigma_{g}\right) \quad j=0,1 .
$$

Corollary 9. Let $G$ be a connected Lie group and $K$ a maximal compact subgroup such that $\operatorname{dim}(G / K)=3$. Let $\Gamma$ be a uniform lattice in $G$ and $\sigma \in H^{2}(\Gamma, U(1))$ be any multiplier on $\Gamma$. If $G$ is $K$-amenable, then

$$
K_{j}\left(C_{r}^{*}(\Gamma, \sigma)\right) \cong K_{j}\left(C_{r}^{*}(\Gamma)\right) \cong K^{j+1}(\Gamma \backslash G / K), \quad \text { for } j=0,1 \quad(\bmod 2) .
$$

Proof. By Theorem 10, we see that

$$
K_{j}\left(C_{r}^{*}(\Gamma)\right) \cong K^{j+\operatorname{dim}(G / K)}(\Gamma \backslash G / K), \quad \text { for } j=0,1 \quad(\bmod 2) .
$$

By the Packer-Raeburn stabilization trick, $C_{r}^{*}(\Gamma, \sigma)$ is Morita equivalent to $\mathcal{K} \rtimes \Gamma$, and because $G$ is $K$-amenable, $\mathcal{K} \rtimes \Gamma \otimes C_{0}(G / K)$ is Morita equivalent to $B_{\sigma}=C\left(\Gamma \backslash G / K, \mathcal{E}_{\sigma}\right)$, where $\mathcal{E}_{\sigma}$ is as before, a locally trivial bundle of $C^{*}$-algebras over $\Gamma \backslash G / K$ with fibre $\mathcal{K}$. Finally, the Dixmier-Douady invariant

$$
\delta\left(B_{\sigma}\right)=\delta(\sigma) \in H^{3}(\Gamma \backslash G / K, \mathbb{Z}) \cong H^{3}(\Gamma, \mathbb{Z}) .
$$

Suppose now that $\Gamma \backslash G / K$ is not orientable. Then $H^{3}(\Gamma \backslash G / K, \mathbb{Z})=\{0\}$ and therefore $\delta\left(B_{\sigma}\right)=\delta(\sigma)=0$. Hence $B_{\sigma}$ is Morita equivalent to $C(\Gamma \backslash G / K)$ and we have $(*)$ in this case.

Suppose next that $\Gamma \backslash G / K$ is orientable. The short exact sequence of coefficient groups

$$
1 \rightarrow \mathbb{Z} \stackrel{i}{\rightarrow} \mathbb{R} \stackrel{e^{2 \pi \sqrt{-1}}}{\longrightarrow} U(1) \rightarrow 1
$$

gives rise to a long exact sequence of cohomology groups (the change of coefficient groups sequence)

$$
\cdots \rightarrow H^{2}(\Gamma, \mathbb{R}) \stackrel{e^{2 \pi \sqrt{-1}} *}{\longrightarrow} H^{2}(\Gamma, U(1)) \stackrel{\delta}{\rightarrow} H^{3}(\Gamma, \mathbb{Z}) \stackrel{i_{*}}{\rightarrow} H^{3}(\Gamma, \mathbb{R}) \rightarrow \cdots
$$

Since $\Gamma \backslash G / K$ is oriented, we see that $H^{3}(\Gamma, \mathbb{Z}) \cong \mathbb{Z}$ and $H^{3}(\Gamma, \mathbb{R}) \cong \mathbb{R}$ are both generated by the fundamental orientation class of $\Gamma \backslash G / K,[\Gamma \backslash G / K]$, and since $i_{*}[\Gamma \backslash G / K]=$ $[\Gamma \backslash G / K]$, we see that $i_{*}$ is injective. Therefore by the exactness of $(* *)$ at $H^{3}(\Gamma, \mathbb{Z})$, one has $\delta(\sigma)=0$ for all $\sigma \in H^{2}(\Gamma, U(1))$, and so we see that $B_{\sigma}$ is Morita equivalent to $C(\Gamma \backslash G / K)$, and again we have $(*)$ in this case.

Corollary 10. Let $M=K(\Gamma, 1)$ be a connected locally-symmetric, compact, 3-dimensional manifold. Let $\sigma \in H^{2}(\Gamma, U(1))$ be any multiplier on $\Gamma$, then one has

$$
K_{j}\left(C_{r}^{*}(\Gamma, \sigma)\right) \cong K_{j}\left(C_{r}^{*}(\Gamma)\right) \cong K^{j+1}(M), \quad j=0,1 .
$$

Proof. Since $M$ is locally symmetric, it is of the form $\Gamma \backslash G / K$, where $G$ is a connected Lie group, $K$ is a maximal compact subgroup such that $\operatorname{dim}(G / K)=3$ and $\Gamma \subset G$ is a uniform lattice in $G$. We need to verify that $\gamma_{G}=1$. According to Thurston's list of 3-dimensional geometries or locally homogeneous spaces, one has 
1. $G=\mathbb{R}^{3} \rtimes \mathbf{S O}(3), G / K=\mathbb{R}^{3}, \gamma_{G}=1$ since $\mathbb{R}^{3}$ and $\mathbf{S O}(3)$ are amenable, and so is their semidirect product.

2. $G=\mathbf{S O}_{0}(3,1), G / K=\mathbb{H}^{3}, \gamma_{G}=1$ by Kasparov's theorem.

3. $G=\mathbf{S O}_{0}(2,1) \rtimes \mathbb{R}, G / K=\mathbb{H}^{2} \times \mathbb{R}, \gamma_{G}=1$ since it's the semidirect product of $K$-amenable groups.

4. $G=$ Heis, $G / K=$ Heis, $\gamma_{G}=1$ since Heis is nilpotent and hence an amenable group.

5. $G=$ Solv, $G / K=$ Solv, $\gamma_{G}=1$ since Solv is a solvable group and hence an amenable group.

6. $G=\mathbf{S} \widetilde{\mathbf{O}_{0}(2,1)} \rtimes \mathbb{R}, G / K=\widetilde{\mathbf{S O}_{0}(2,1)}$. Firstly, $\gamma_{\mathbf{S O}_{0}(2,1)}=1$ since $\widetilde{\mathbf{S O}_{0}(2,1)}$ is the semidirect product of the $K$-amenable groups $\mathbf{S O}_{0}(2,1)$ and $\mathbb{Z}$. Also $\gamma_{G}=1$, since its the semidirect product of the $K$-amenable groups $\widehat{\mathbf{S O}_{0}(2,1)}$ and $\mathbb{R}$.

The other two locally homogeneous spaces in Thurston's list are not locally symmetric. We now apply Corollary 9 to deduce Corollary 10.

An interesting question is whether Corollary 10 is true without the locally symmetric assumption on $M$. We formulate this in terms of a conjecture.

Conjecture. Let $M=K(\Gamma, 1)$ be a connected, compact, 3-dimensional manifold which is an Eilenberg-Maclane space with fundamental group $\Gamma$. Then for any multiplier $\sigma \in$ $H^{2}(\Gamma, U(1))$ on $\Gamma$, one has

$$
K_{j}\left(C_{r}^{*}(\Gamma, \sigma)\right) \cong K_{j}\left(C_{r}^{*}(\Gamma)\right) \cong K^{j+1}(M), \quad j=0,1 .
$$

Remarks. Selected portions of our proof of Corollary 9 go through in the situation described in the conjecture. More precisely, the proof of Corollary 9 shows that the DixmierDouady invariant $\delta(\sigma)=0$ for all $\sigma \in H^{2}(\Gamma, U(1))$ for $\Gamma$ as in the conjecture.

\section{Range of the trace And the Kadison COnstant}

In this section, we will prove some structural theorems for the twisted group $C^{*}$-algebras that are relevant to the 'Martini' problems described in the introduction. The first of these calculates the range of the canonical trace map on $K_{0}$ of the twisted group $C^{*}$-algebras. We use in an essential way the results of the previous section as well as a twisted version of the $L^{2}$-index theorem of Atiyah [At], which is due to Gromov Gr2. This enables us to deduce information about projections in the twisted group $C^{*}$-algebras. In the case of no twisting, this follows because the Baum-Connes conjecture is known to be true while these results are also well known for the case of the irrational rotation algebras. However, our approach here is novel, and as we will show elsewhere $\mathrm{Ma}$, enables a generalization of most of the known results.

9.1. Twisted Kasparov map. Suppose that $\Gamma_{g}$ is a discrete, cocompact subgroup of $\mathbf{S O}_{0}(2,1)$ that is, $\Gamma_{g}$ is the fundamental group of Riemann surface $\Sigma_{g}$ of genus $g>1$. 
Then for any $\sigma \in H^{2}\left(\Gamma_{g}, U(1)\right)$, the twisted Kasparov isomorphism,

$$
\mu_{\sigma}: K_{\bullet}\left(\Sigma_{g}\right) \rightarrow K_{\bullet}\left(C_{r}^{*}\left(\Gamma_{g}, \sigma\right)\right)
$$

is defined as follows. Here $K_{0}\left(\Sigma_{g}\right)$ denotes the $K$-homology group of $\Sigma_{g}$. Since $\Sigma_{g}$ is spin, it is $K$-oriented and by Poincaré duality, the $K$ groups $K^{j}\left(\Sigma_{g}\right)$ are naturally isomorphic to the corresponding $K$-homology groups $K_{j}\left(\Sigma_{g}\right)$ for $j=0,1$. Explicitly, let $\mathcal{E} \rightarrow \Sigma_{g}$ be a vector bundle over $\Sigma_{g}$ defining an element $[\mathcal{E}]$ in $K^{0}\left(\Sigma_{g}\right)$. Under Poincaré duality, $[\mathcal{E}]$ corresponds to the twisted Dirac operator $\phi_{\mathcal{E}}^{+}: L^{2}\left(\Sigma_{g}, \mathcal{S}^{+} \otimes \mathcal{E}\right) \rightarrow L^{2}\left(\Sigma_{g}, \mathcal{S}^{-} \otimes \mathcal{E}\right)$ where $\mathcal{S}^{ \pm}$denote the $\frac{1}{2}$ spinor bundles over $\Sigma_{g}$. That is,

$$
\begin{aligned}
P D: K^{0}\left(\Sigma_{g}\right) & \rightarrow K_{0}\left(\Sigma_{g}\right) \\
{[\mathcal{E}] } & \rightarrow\left[\not_{\mathcal{E}}^{+}\right]
\end{aligned}
$$

is the Poincaré duality isomorphism. By Corollary 8 of the previous section, there is a canonical isomorphism

$$
K_{\bullet}\left(C_{r}^{*}\left(\Gamma_{g}, \sigma\right)\right) \cong K^{\bullet}\left(\Sigma_{g}\right) .
$$

Both of these maps are assembled to yield the twisted Kasparov map as in $\left(^{*}\right)$.

We next describe this map more explicitly. Given $\left[\not \partial \mathcal{E}_{\mathcal{E}}^{+}\right] \in K_{0}\left(\Sigma_{g}\right)$ as above, the lift of this operator to $\mathbb{H}=\widetilde{\Sigma}_{g}$, the universal cover of $\Sigma_{g}$,

$$
\widetilde{\not_{\mathcal{E}}^{+}}: L^{2}\left(\mathbb{H}, \widetilde{\mathcal{S}^{+} \otimes \mathcal{E}}\right) \rightarrow L^{2}\left(\mathbb{H}, \widetilde{\mathcal{S}^{-} \otimes \mathcal{E}}\right)
$$

is a $\Gamma_{g}$-invariant operator. Consider now the short exact sequence of coefficient groups

$$
1 \rightarrow \mathbb{Z} \stackrel{i}{\rightarrow} \mathbb{R} \stackrel{e^{2 \pi \sqrt{-1}}}{\longrightarrow} U(1) \rightarrow 1
$$

which gives rise to a long exact sequence of cohomology groups (the change of coefficient groups sequence)

$$
\cdots \rightarrow H^{2}\left(\Gamma_{g}, \mathbb{Z}\right) \stackrel{i_{*}}{\rightarrow} H^{2}\left(\Gamma_{g}, \mathbb{R}\right) \stackrel{e^{2 \pi \sqrt{-1}}}{\longrightarrow} H^{2}\left(\Gamma_{g}, U(1)\right) \stackrel{\delta}{\rightarrow} 0 .
$$

Therefore for any multiplier $\sigma \in H^{2}\left(\Gamma_{g}, U(1)\right)$ of $\Gamma_{g}$, there is a 2-form $\omega$ on $\Sigma_{g}$ such that $e^{2 \pi \sqrt{-1}}([\omega])=\sigma$. Of course, the choice of $\omega$ is not unique, but this will not affect the results that we are concerned with. Let $\widetilde{\omega}$ denote the lift of $\omega$ to the universal cover $\mathbb{H}$. Since the hyperbolic plane $\mathbb{H}$ is contractible, it follows that $\widetilde{\omega}=d \eta$ where $\eta$ is a 1 -form on $\mathbb{H}$ which is not in general $\Gamma_{g}$ invariant. Now let $\nabla=d-i \eta$ denote a connection on the trivial complex line bundle on $\mathbb{H}$. Note that the curvature of $\nabla$ is $\nabla^{2}=i \omega$. Consider now the operator

$$
\widetilde{\not_{\mathcal{E}}^{+}} \otimes \nabla: L^{2}\left(\mathbb{H}, \widetilde{\mathcal{S}^{+} \otimes \mathcal{E}}\right) \rightarrow L^{2}\left(\mathbb{H}, \widetilde{\mathcal{S}^{-} \otimes \mathcal{E}}\right) .
$$

It does not commute with the $\Gamma_{g}$ action, but it does commute with the projective action of $\Gamma_{g}$ which is defined by the multiplier $\sigma$, and by a mild generalization of the index theorem of $[\mathrm{CM}]$, it has a $\Gamma_{g^{-}} L^{2}$-index

$$
\operatorname{ind}_{\Gamma_{g}}\left(\widetilde{\partial_{\mathcal{E}}^{+}} \otimes \nabla\right) \in K_{0}\left(\mathbb{C}\left(\Gamma_{g}, \sigma\right) \otimes \mathcal{R}\right)
$$


where $\mathcal{R}$ denotes the algebra of smoothing operators. Then observe that the twisted Kasparov map is merely

$$
\mu_{\sigma}\left(\left[\not_{\mathcal{E}^{+}}\right]\right)=j_{*}\left(\operatorname{ind}_{\Gamma_{g}}\left(\widetilde{\not_{\mathcal{E}}^{+}} \otimes \nabla\right)\right) \in K_{0}\left(C^{*}\left(\Gamma_{g}, \sigma\right)\right),
$$

where $j: \mathbb{C}\left(\Gamma_{g}, \sigma\right) \otimes \mathcal{R} \rightarrow C_{r}^{*}\left(\Gamma_{g}, \sigma\right) \otimes \mathcal{K}$ is the natural inclusion map, and

$$
j_{*}: K_{0}\left(\mathbb{C}\left(\Gamma_{g}, \sigma\right) \otimes \mathcal{R}\right) \rightarrow K_{0}\left(C_{r}^{*}\left(\Gamma_{g}, \sigma\right)\right)
$$

is the induced map on $K_{0}$.

The canonical trace on $\left.C_{r}^{*}\left(\Gamma_{g}, \sigma\right)\right)$ induces a linear map

$$
[\operatorname{tr}]: K_{0}\left(C_{r}^{*}\left(\Gamma_{g}, \sigma\right)\right) \rightarrow \mathbb{R}
$$

which is called the trace map in $K$-theory. Explicitly, first tr extends to matrices with entries in $C^{*}\left(\Gamma_{g}, \sigma\right)$ as (with Trace denoting matrix trace):

$$
\operatorname{tr}(f \otimes r)=\operatorname{Trace}(r) \operatorname{tr}(f) .
$$

Then the extension of tr to $K_{0}$ is given by $[\operatorname{tr}]([e]-[f])=\operatorname{tr}(e)-\operatorname{tr}(f)$, where $e, f$ are idempotent matrices with entries in $\left.C^{*}\left(\Gamma_{g}, \sigma\right)\right)$.

9.2. The isomorphism classes of algebras $C^{*}\left(\Gamma_{g}, \sigma\right)$. Let $\sigma \in Z^{2}\left(\Gamma_{g}, U(1)\right)$ be a multiplier on $\Gamma_{g}$. If $\sigma^{\prime} \in Z^{2}\left(\Gamma_{g}, U(1)\right)$ is another multiplier on $\Gamma_{g}$ such that $[\sigma]=\left[\sigma^{\prime}\right] \in$ $H^{2}\left(\Gamma_{g}, U(1)\right)$, then it can be easily shown that $C^{*}\left(\Gamma_{g}, \sigma\right) \cong C^{*}\left(\Gamma_{g}, \sigma^{\prime}\right)$. That is, the isomorphism classes of the $C^{*}$-algebras $C^{*}\left(\Gamma_{g}, \sigma\right)$ are naturally parametrized by $H^{2}\left(\Gamma_{g}, U(1)\right)$. But $H^{2}\left(\Gamma_{g}, U(1)\right) \cong H^{2}\left(\Sigma_{g}, U(1)\right) \cong U(1)$ and the isomorphism is given explicitly by $[\sigma] \rightarrow<[\sigma],\left[\Sigma_{g}\right]>$, where $[\sigma]$ is now viewed as a Cech 2-cocycle on $\Sigma_{g}$ with coefficients in $U(1)$, and $\left[\Sigma_{g}\right]$ denotes the fundamental class of the genus $g$ Riemann surface. We summarize this below.

Lemma 13. The isomorphism classes of twisted group $C^{*}$-algebras $C^{*}\left(\Gamma_{g}, \sigma\right)$ are naturally parametrized by $U(1) \cong \mathbb{R} / \mathbb{Z} \cong(0,1]$. The classification map is given explicitly by

$$
[\sigma] \rightarrow<[\sigma],\left[\Sigma_{g}\right]>
$$

where $[\sigma]$ is now viewed as a Čech 2-cocycle on $\Sigma_{g}$ with coefficients in $U(1)$, and $\left[\Sigma_{g}\right]$ denotes the fundamental class of the genus $g$ Riemann surface.

9.3. Range of the trace map on $K_{0}$. We can now state the first major theorem of this section.

Theorem 12. The range of the trace map is

$$
[\operatorname{tr}]\left(K_{0}\left(C_{r}^{*}\left(\Gamma_{g}, \sigma\right)\right)\right)=\mathbb{Z} \theta+\mathbb{Z},
$$

where $2 \pi \theta=<\sigma,\left[\Sigma_{g}\right]>\in(0,1]$ is the result of pairing the multiplier $\sigma$ on $\Gamma_{g}$ with the fundamental class of $\Sigma_{g}$. 
Proof. We first observe that by the results of the previous section the twisted Kasparov map is an isomorphism. Therefore to compute the range of the trace map on $K_{0}$, it suffices to compute the range of the trace map on elements of the form

$$
\mu_{\sigma}\left(\left[\not_{\mathcal{E}^{0}}^{+}\right]-\left[\not_{\mathcal{E}^{1}}^{+}\right]\right)
$$

for any element

$$
\left[\not_{\mathcal{E}^{0}}^{+}\right]-\left[\not_{\mathcal{E}^{1}}^{+}\right] \in K_{0}\left(\Sigma_{g}\right) .
$$

By the twisted analogue of the $L^{2}$ index theorem of Atiyah [At] and Singer [Si] for elliptic operators on a covering space that are invariant under the projective action of the fundamental group defined by $\sigma$, and which is due to Gromov [Gr2] (see also [Ma for a detailed proof of a further generalization), one has

$$
[\tau]\left(\operatorname{ind}_{\Gamma_{g}}\left(\widetilde{\partial_{\mathcal{E}}^{+}} \otimes \nabla\right)\right)=\frac{1}{2 \pi}\left\langle\hat{A}\left(\Sigma_{g}\right) \operatorname{ch}(\mathcal{E}) e^{[\omega]},\left[\Sigma_{g}\right]\right\rangle .
$$

We next simplify the right hand side of $(*)$ using

$$
\begin{aligned}
\hat{A}\left(\Sigma_{g}\right) & =1 \\
\operatorname{ch}(\mathcal{E}) & =\operatorname{rank} \mathcal{E}+c_{1}(\mathcal{E}) \\
e^{[\omega]} & =1+[\omega] .
\end{aligned}
$$

Therefore one has

$$
[\tau]\left(\operatorname{ind}_{\Gamma_{g}}\left(\widetilde{\not_{\mathcal{E}}^{+}} \otimes \nabla\right)\right)=\operatorname{rank} \mathcal{E} \frac{\left\langle[\omega],\left[\Sigma_{g}\right]\right\rangle}{2 \pi}+\frac{\left\langle c_{1}(\mathcal{E}),\left[\Sigma_{g}\right]\right\rangle}{2 \pi},
$$

and we see that

$$
[\tau]\left(\mu_{\sigma}\left(\left[\not_{\mathcal{E}^{0}}^{+}\right]-\left[\not_{\mathcal{E}^{1}}^{+}\right]\right)\right)=\left(\operatorname{rank} \mathcal{E}^{0}-\operatorname{rank} \mathcal{E}^{1}\right) \frac{\left\langle[\omega],\left[\Sigma_{g}\right]\right\rangle}{2 \pi}+\frac{\left\langle c_{1}\left(\mathcal{E}^{0}\right)-c_{1}\left(\mathcal{E}^{1}\right),\left[\Sigma_{g}\right]\right\rangle}{2 \pi}
$$

It follows that the range of the trace map on $K_{0}$ is $\mathbb{Z} \frac{\left\langle[\omega],\left[\Sigma_{g}\right]\right\rangle}{2 \pi}+\mathbb{Z}=\mathbb{Z} \theta+\mathbb{Z}$, because

$$
\frac{\left\langle[\omega],\left[\Sigma_{g}\right]\right\rangle}{2 \pi}-\theta \in \mathbb{Z}
$$

We will now discuss some applications of this result. We begin by studying projections in the twisted group $C^{*}$-algebra, which is a problem of independent interest.

Proposition 4. Let $\sigma \in H^{2}\left(\Sigma_{g}, \mathbb{R} / \mathbb{Z}\right)$ be a multiplier on $\Gamma_{g}$, and $2 \pi \theta=<\sigma,\left[\Sigma_{g}\right]>\in(0,1]$ be the result of pairing $\sigma$ with the fundamental class of $\Sigma_{g}$. If $\theta=p / q$ is rational, then there are only $q-1$ unitary equivalence classes of projections, other than 0 and 1 , in the reduced twisted group $C^{*}$-algebra $C_{r}^{*}\left(\Gamma_{g}, \sigma\right)$.

Proof. By assumption, $\theta=p / q$. Let $P$ be a projection in $C_{r}^{*}\left(\Gamma_{g}, \sigma\right)$. Then $1-P$ is also a projection in $C_{r}^{*}\left(\Gamma_{g}, \sigma\right)$ and one has

$$
1=\operatorname{tr}(1)=\operatorname{tr}(P)+\operatorname{tr}(1-P) .
$$

Each term in the above equation is non-negative. By the previous theorem, it follows that $\operatorname{tr}(P) \in\{0,1 / q, 2 / q, \ldots 1\}$. By faithfulness and normality of the trace tr, it follows that 
there are only $q-1$ unitary equivalence classes of projections, other than those of 0 and 1 in $C_{r}^{*}\left(\Gamma_{g}, \sigma\right)$.

Our second application will involve the Kadison constant of a twisted group $C^{*}$-algebra, which we will now recall. The Kadison constant of $C_{r}^{*}\left(\Gamma_{g}, \sigma\right)$ is defined by:

$$
C_{\sigma}\left(\Gamma_{g}\right)=\inf \left\{\operatorname{tr}(P): P \text { is a non-zero projection in } C_{r}^{*}\left(\Gamma_{g}, \sigma\right) \otimes \mathcal{K}\right\} .
$$

Recall from earlier sections the following Hamiltonians:

$$
H_{\eta}=(d-i \eta)^{*}(d-i \eta)=\nabla^{*} \nabla
$$

and

$$
H_{\eta, V}=H_{\eta}+V
$$

where $V$ is any $\Gamma_{g}$-invariant potential on $\mathbb{H}$. The operators $H_{\eta}$ and $H_{\eta, V}$ are invariant under the projective $\left(\Gamma_{g}, \sigma\right)$-action.

Proposition 5. Let $\sigma \in H^{2}\left(\Sigma_{g}, \mathbb{R} / \mathbb{Z}\right)$ be a multiplier on $\Gamma_{g}$, and $2 \pi \theta=<\sigma,\left[\Sigma_{g}\right]>\in(0,1]$ be the result of pairing $\sigma$ with the fundamental class of $\Sigma_{g}$. If $\theta=p / q$ is rational, then the spectrum of any associated Hamiltonian $H_{\eta, V}$ has a band structure, in the sense that the intersection of the resolvent set with any compact interval in $\mathbb{R}$ has only a finite number of components. In particular, the intersection of $\sigma\left(H_{\eta, V}\right)$ with any compact interval in $\mathbb{R}$ is never a Cantor set.

Proof. By the previous proposition, it follows that one has the estimate $C_{\sigma}\left(\Gamma_{g}\right) \geq 1 / q>0$. Then one applies the main result in Brüning-Sunada [BrSu] to deduce the proposition.

This leaves open the question of whether there are Hamiltonians with Cantor spectrum when $\theta$ is irrational. In the Euclidean case, this is usually known as the Ten Martini Problem, and is to date, not completely solved, though much progress has been made (cf. [Sh]). We pose a generalization of this problem to the hyperbolic case (which also includes the Euclidean case):

Conjecture (The Ten Dry Martini Problem). Let $\sigma \in H^{2}\left(\Sigma_{g}, \mathbb{R} / \mathbb{Z}\right)$ be a multiplier on $\Gamma_{g}$, and $2 \pi \theta=<\sigma,\left[\Sigma_{g}\right]>\in(0,1]$ be the result of pairing $\sigma$ with the fundamental class of $\Sigma_{g}$. If $\theta$ is irrational, then there is an associated Hamiltonian $H_{\eta, V}$ with a Cantor set type spectrum, in the sense that the intersection of $\sigma\left(H_{\eta, V}\right)$ with some compact interval in $\mathbb{R}$ is a Cantor set.

We will next apply the range of the trace Theorem 12 to deduce results about the discrete Hamiltonian $H_{\tau}$, as in Section 9.

Proposition 6. Let $\sigma \in H^{2}\left(\Sigma_{g}, \mathbb{R} / \mathbb{Z}\right)$ be a multiplier on $\Gamma_{g}$, and $2 \pi \theta=<\sigma,\left[\Sigma_{g}\right]>\in(0,1]$ be the result of pairing $\sigma$ with the fundamental class of $\Sigma_{g}$. If $\theta=p / q$ is rational, then the spectrum of the associated discrete Hamiltonian $H_{\tau}$ has a band structure, in the sense that the intersection of the resolvent set with $\mathbb{R}$ has only a finite number of components. In particular, the intersection of $\sigma\left(H_{\tau}\right)$ with any compact interval in $\mathbb{R}$ is never a Cantor set. 
Proof. From the estimate $C_{\sigma}\left(\Gamma_{g}\right) \geq 1 / q>0$ the main result in Sun implies the proposition.

This leads us to our next conjecture.

Conjecture (The Discrete Ten Dry Martini Problem). Let $\sigma \in H^{2}\left(\Sigma_{g}, \mathbb{R} / \mathbb{Z}\right)$ be a multiplier on $\Gamma_{g}$, and $2 \pi \theta=<\sigma,\left[\Sigma_{g}\right]>\in(0,1]$ be the result of pairing $\sigma$ with the fundamental class of $\Sigma_{g}$. If $\theta$ is irrational, then the associated Hamiltonian $H_{\tau}$ has Cantor spectrum.

9.4. On the classification of twisted group $C^{*}$-algebras. We will now use the range of the trace Theorem 12, to give a complete classification, up to isomorphism, of the twisted group $C^{*}$-algebras $C^{*}(\Gamma, \sigma)$. A similar complete classification, up to Morita equivalence, is contained in Ma.

Proposition 7 (Isomorphism classification of twisted group $C^{*}$-algebras). Let $\sigma, \sigma^{\prime} \in$ $H^{2}\left(\Sigma_{g}, \mathbb{R} / \mathbb{Z}\right)$ be multipliers on $\Gamma_{g}$, and $2 \pi \theta=<\sigma,\left[\Sigma_{g}\right]>\in(0,1], 2 \pi \theta^{\prime}=<\sigma^{\prime},\left[\Sigma_{g}\right]>\in(0,1]$ be the result of pairing $\sigma, \sigma^{\prime}$ with the fundamental class of $\Sigma_{g}$. Then $C^{*}\left(\Gamma_{g}, \sigma\right) \cong C^{*}\left(\Gamma_{g}, \sigma^{\prime}\right)$ if and only if $\theta^{\prime} \in\{\theta, 1-\theta\}$.

Proof. Let tr and $\operatorname{tr}^{\prime}$ denote the canonical traces on $C^{*}\left(\Gamma_{g}, \sigma\right)$ and $C^{*}\left(\Gamma_{g}, \sigma^{\prime}\right)$ respectively. Let

be an isomorphism, and let

$$
\phi: C^{*}\left(\Gamma_{g}, \sigma\right) \cong C^{*}\left(\Gamma_{g}, \sigma^{\prime}\right)
$$

$$
\phi_{*}: K_{0}\left(C^{*}\left(\Gamma_{g}, \sigma\right)\right) \cong K_{0}\left(C^{*}\left(\Gamma_{g}, \sigma^{\prime}\right)\right)
$$

denote the induced map on $K_{0}$. By Theorem 12, the range of the trace map on $K_{0}$ is

$$
[\operatorname{tr}]\left(K_{0}\left(C^{*}\left(\Gamma_{g}, \sigma\right)\right)\right)=\mathbb{Z} \theta+\mathbb{Z}
$$

and

$$
\left[\operatorname{tr}^{\prime}\right]\left(K_{0}\left(C^{*}\left(\Gamma_{g}, \sigma^{\prime}\right)\right)\right)=\mathbb{Z} \theta^{\prime}+\mathbb{Z}
$$

So there are elements $[P] \in K_{0}\left(C^{*}\left(\Gamma_{g}, \sigma\right)\right)$ and $\left[P^{\prime}\right] \in K_{0}\left(C^{*}\left(\Gamma_{g}, \sigma^{\prime}\right)\right)$ such that $[\operatorname{tr}]([P])=$ $\theta$ and $\left[\operatorname{tr}^{\prime}\right]\left(\left[P^{\prime}\right]\right)=\theta^{\prime}$. Clearly one has $\operatorname{tr} \circ \phi=\operatorname{tr}^{\prime}$, which induces the identity $[\operatorname{tr}] \circ$ $\phi_{*}=\left[\operatorname{tr}^{\prime}\right]$ in $K_{0}\left(C^{*}\left(\Gamma_{g}, \sigma^{\prime}\right)\right)$. In Section 1 , we have proved that $K_{0}\left(C^{*}\left(\Gamma_{g}, \sigma\right)\right) \cong \mathbb{Z}^{2} \cong$ $K_{0}\left(C^{*}\left(\Gamma_{g}, \sigma^{\prime}\right)\right)$. In the basis above, one has

$$
\phi_{*}: \mathbb{Z}[P] \oplus \mathbb{Z} \cong K_{0}\left(C^{*}\left(\Gamma_{g}, \sigma\right)\right) \rightarrow K_{0}\left(C^{*}\left(\Gamma_{g}, \sigma^{\prime}\right)\right) \cong \mathbb{Z}\left[P^{\prime}\right] \oplus \mathbb{Z} .
$$

Since $\phi_{*}[1]=[1]$ and $\phi_{*} \in \mathbf{G L}(2, \mathbb{Z})$, one sees that there is an integer $n$ such that

$$
\phi_{*}=\left(\begin{array}{cc}
1 & n \\
0 & \pm 1
\end{array}\right) .
$$

Assembling these results, one has $\theta=[\operatorname{tr}]([P])=[\operatorname{tr}]\left(\phi_{*}[P]\right)=\left[\operatorname{tr}^{\prime}\right]\left(n[1] \pm\left[P^{\prime}\right]\right)=n \pm \theta^{\prime}$. Since $\theta, \theta^{\prime} \in(0,1]$, one deduces that $\theta^{\prime} \in\{\theta, 1-\theta\}$.

Let $\psi: \Sigma_{g} \rightarrow \Sigma_{g}$ be an orientation reversing diffeomorphism. We can assume without loss of generality that $\psi$ has a fixed point $x_{0} \in \Sigma_{g}$. This is because there is an orientation preserving diffeomorphism $\eta$ of $\Sigma_{g}$ whose value at the point $\psi\left(x_{0}\right)$ is equal to $x_{0}$; in fact 
$\eta$ can be chosen to be isotopic to the identity (cf. exercise A2, chapter 1, [Helg]). The composition $\eta \circ \psi$ is then an orientation reversing diffeomorphism of $\Sigma_{g}$ with fixed point $x_{0}$. Then $\psi$ induces an automorphism $\psi_{*}: \Gamma_{g} \rightarrow \Gamma_{g}$ of the fundamental group $\pi_{1}\left(\Sigma_{g}, x_{0}\right) \cong \Gamma_{g}$. We first evaluate $\left\langle\psi^{*} \sigma,\left[\Sigma_{g}\right]>=<\sigma, \psi_{*}\left[\Sigma_{g}\right]\right\rangle=\overline{\left\langle\sigma,\left[\Sigma_{g}\right]\right\rangle}=\left\langle\bar{\sigma},\left[\Sigma_{g}\right]\right\rangle$, since $\psi$ is orientation reversing. By Lemma 13 we see that $\psi^{*} \sigma=\bar{\sigma} \in H^{2}\left(\Gamma_{g}, U(1)\right)$. Therefore the automorphism $\psi_{*}$ of $\Gamma_{g}$ induces an isomorphism of twisted group $C^{*}$-algebras

$$
C^{*}\left(\Gamma_{g}, \sigma\right) \cong C^{*}\left(\Gamma_{g}, \psi^{*} \sigma\right) \cong C^{*}\left(\Gamma_{g}, \bar{\sigma}\right) .
$$

Therefore if $\theta^{\prime} \in\{\theta, 1-\theta\}$, one has $C^{*}\left(\Gamma_{g}, \sigma\right) \cong C^{*}\left(\Gamma_{g}, \sigma^{\prime}\right)$, completing the proof of the proposition.

9.5. Twisted $\mathbf{I C C}$ group von Neumann algebras and type $\mathbf{I I}_{1}$ factors. Recall that an ICC group $\Gamma$ is one in which every non-trivial conjugacy class is infinite. There are many examples of ICC groups, such as free groups, fundamental groups of compact surfaces etc. It is well known that the group von Neumann algebras of these groups are type $\mathrm{II}_{1}$ factors Tak. We will now prove that a similar result holds for the twisted group von Neumann algebras (this result probably exists in the literature but for completeness we reproduce a proof). We briefly recall some definitions. Let $W^{*}(\Gamma, \sigma)$ denote the twisted group von Neumann algebra, where $\sigma$ is a multiplier on $\Gamma$, which is by definition the weak closure of $C^{*}(\Gamma, \sigma)$, or equivalently, the weak closure of the algebraic group algebra $\mathbb{C}(\Gamma, \sigma)$ in the $\sigma$-regular representation on $\ell^{2}(\Gamma)$. Let $\operatorname{Proj}\left(W^{*}(\Gamma, \sigma)\right)$ denote the set of all projections in $W^{*}(\Gamma, \sigma)$. Then one has.

Proposition 8. Let $\Gamma$ be an ICC group, and $\sigma \in H^{2}(\Sigma, \mathbb{R} / \mathbb{Z})$ be a multiplier on $\Gamma$. Then $W^{*}(\Gamma, \sigma)$ is a $I I_{1}$ factor. In particular, $\operatorname{tr}\left(\operatorname{Proj}\left(W^{*}(\Gamma, \sigma)\right)\right)=[0,1]$.

Proof. By the commutant theorem for the regular $\sigma$-representation we see that the commutant of $W^{*}(\Gamma, \sigma)$ is identified with $W^{*}(\Gamma, \bar{\sigma})$. We need to compute the centre $Z(\Gamma, \sigma)$ of $W^{*}(\Gamma, \sigma)$, which is equal to the intersection $Z(\Gamma, \sigma)=W^{*}(\Gamma, \sigma) \cap W^{*}(\Gamma, \bar{\sigma})$. Let $T: \Gamma \rightarrow B\left(\ell^{2}(\Gamma)\right)$ denote the left projective $(\Gamma, \sigma)$-action. Regard $x \in W^{*}(\Gamma, \bar{\sigma})$ as $x=\sum_{\gamma \in \Gamma} x(\gamma) T(\gamma)$. Since $W^{*}(\Gamma, \sigma)$ is the weak closure of $\mathbb{C}(\Gamma, \sigma)$, it follows that $(x(\gamma))_{\gamma \in \Gamma} \in \ell^{2}(\Gamma)$. Now $x \in Z(\Gamma, \sigma)$ if and only if $x$ commutes with $T\left(\gamma^{\prime}\right), \gamma^{\prime} \in \Gamma$. But

$$
\begin{aligned}
T\left(\gamma^{\prime}\right) x & =\sum_{\gamma \in \Gamma} x(\gamma) \sigma\left(\gamma^{\prime}, \gamma\right) T\left(\gamma^{\prime} \gamma\right) \\
& =\sum_{\gamma \in \Gamma} x\left(\gamma^{\prime-1} \gamma\right) \sigma\left(\gamma^{\prime}, \gamma^{\prime-1} \gamma\right) T(\gamma)
\end{aligned}
$$

and

$$
\begin{aligned}
x T\left(\gamma^{\prime}\right) & =\sum_{\gamma \in \Gamma} x(\gamma) \sigma\left(\gamma, \gamma^{\prime}\right) T\left(\gamma \gamma^{\prime}\right) \\
& =\sum_{\gamma \in \Gamma} x\left(\gamma \gamma^{\prime-1}\right) \sigma\left(\gamma \gamma^{\prime-1}, \gamma^{\prime}\right) T(\gamma) .
\end{aligned}
$$


Therefore we see that $x\left(\gamma^{\prime^{-1}} \gamma\right) \sigma\left(\gamma^{\prime}, \gamma^{\prime-1} \gamma\right)=x\left(\gamma \gamma^{\prime^{-1}}\right) \sigma\left(\gamma \gamma^{\prime^{-1}}, \gamma^{\prime}\right)$ for all $\gamma^{\prime} \in \Gamma$. So $\left|x\left(\gamma^{\prime-1} \gamma \gamma^{\prime}\right)\right|=|x(\gamma)|$ for all $\gamma^{\prime} \in \Gamma$. That is, $|x(\cdot)|$ is constant on each conjugacy class. Now since $x \in \ell^{2}(\Gamma)$, it follows that $x$ vanishes on each infinite conjugacy class. Since $\Gamma$ is an ICC group, it follows that $x(\gamma)=0$ for all $\gamma \neq 1$, that is $Z(\Gamma, \sigma)$ is 1 -dimensional and $W^{*}(\Gamma, \sigma)$ is a $\mathrm{II}_{1}$ factor.

\section{The TOPOLOGICAL INDEX AND THE INDEX THEOREM}

This section identifies the Hall conductivity $\tau_{c}(P, P, P)=\tau(P d P d P)$ with a topological invariant, generalising the work of Xia. Suppose that $\Gamma_{g}$ is a discrete, cocompact subgroup of $\mathbf{S O}_{0}(2,1)$. That is, $\Gamma_{g}$ is the fundamental group of a Riemann surface $\Sigma_{g}$ of genus $g>1$. Then for any $\sigma \in H^{2}\left(\Gamma_{g}, U(1)\right)$, the twisted Kasparov isomorphism,

$$
\mu_{\sigma}: K_{\bullet}\left(\Sigma_{g}\right) \rightarrow K_{\bullet}\left(C_{r}^{*}\left(\Gamma_{g}, \sigma\right)\right)
$$

is defined as in the previous section. We note in the following section (using a result of [Ji]) that given any projection $P$ in $C_{r}^{*}(\Gamma, \sigma)$ there is both a projection $\tilde{P}$ in the same $K_{0}$ class but lying in a dense subalgebra, stable under the holomorphic functional calculus, and a Fredholm module for this dense subalgebra, which may be be paired with $\tilde{P}$ to obtain an analytic index. On the other hand, by the results of the current section, given any such projection $P$ there is a topological index that we can associate to it. The main result we prove here is that the (analytic index) $=$ (topological index).

The first step in the proof is to show that given an additive group cocycle $c \in Z^{2}\left(\Gamma_{g}\right)$ we may define canonical pairings with $K_{0}\left(\Sigma_{g}\right)$ and $K_{0}\left(C_{r}^{*}\left(\Gamma_{g}, \sigma\right)\right)$ which are related by the twisted Kasparov isomorphism, by generalizing some of the results of Connes and Connes-Moscovici to the twisted case. The group 2-cocycle $c$ may be regarded as a skew symmetrised function on $\Gamma_{g} \times \Gamma_{g} \times \Gamma_{g}$, so that we can modify a standard construction in [CM] to obtain a cyclic 2-cocycle $\tau_{c}$ on $\mathbb{C}\left(\Gamma_{g}, \sigma\right) \otimes \mathcal{R}$ by defining:

$$
\tau_{c}\left(f^{0} \otimes r^{0}, f^{1} \otimes r^{1}, f^{2} \otimes r^{2}\right)=\operatorname{Tr}\left(r^{0} r^{1} r^{2}\right) \sum_{g_{0} g_{1} g_{2}=1} f^{0}\left(g_{0}\right) f^{1}\left(g_{1}\right) f^{2}\left(g_{2}\right) c\left(1, g_{1}, g_{1} g_{2}\right) \sigma\left(g_{1}, g_{2}\right) .
$$

Note that $\tau_{c}$ extends to $\mathbb{C}\left(\Gamma_{g}, \sigma\right) \otimes \mathcal{L}^{2}$, (where $\mathcal{L}^{2}$ denotes Hilbert-Schmidt operators) and by the pairing theory of $\mathrm{Co}$ one gets an additive map

$$
\left[\tau_{c}\right]: K_{0}\left(\mathbb{C}\left(\Gamma_{g}, \sigma\right) \otimes \mathcal{R}\right) \rightarrow \mathbb{R}
$$

Explicitly, $\left[\tau_{c}\right]([e]-[f])=\widetilde{\tau}_{c}(e, \cdots, e)-\widetilde{\tau}_{c}(f, \cdots, f)$, where $e, f$ are idempotent matrices with entries in $\left(\mathbb{C}\left(\Gamma_{g}, \sigma\right) \otimes \mathcal{R}\right)^{\sim}=$ unital algebra obtained by adding the identity to $\mathbb{C}\left(\Gamma_{g}, \sigma\right) \otimes \mathcal{R}$ and $\widetilde{\tau}_{c}$ denotes the canonical extension of $\tau_{c}$ to $\left(\mathbb{C}\left(\Gamma_{g}, \sigma\right) \otimes \mathcal{R}\right)^{\sim}$. Let $\widetilde{\not_{\mathcal{E}}^{+}} \otimes \nabla$ be the Dirac operator defined in the previous section, which is invariant under the projective action of the fundamental group defined by $\sigma$. By definition, the $\left(c, \Gamma_{g}, \sigma\right)$-index of $\widetilde{\not_{\mathcal{E}}^{+}} \otimes \nabla$ is

$$
\left[\tau_{c}\right]\left(\operatorname{ind}_{\Gamma_{g}}\left(\widetilde{\partial_{\mathcal{E}}^{+}} \otimes \nabla\right)\right) \in \mathbb{R} .
$$

It only depends on the cohomology class $[c] \in H^{2}\left(\Gamma_{g}\right)$, and it is linear with respect to $[c]$. We assemble this to give the following theorem. 
Theorem 13. Given $[c] \in H^{2}\left(\Gamma_{g}\right)$ and $\sigma \in H^{2}\left(\Gamma_{g}, U(1)\right)$ a multiplier on $\Gamma_{g}$, there is a canonical additive map

$$
\langle[c],\rangle: K_{0}\left(\Sigma_{g}\right) \rightarrow \mathbb{R}
$$

which is defined as

$$
\left\langle[c],\left[\not_{\mathcal{E}}^{+}\right]\right\rangle=\left[\tau_{c}\right]\left(\operatorname{ind}_{\Gamma_{g}}\left(\widetilde{\partial_{\mathcal{E}}^{+}} \otimes \nabla\right)\right) \in \mathbb{R} .
$$

Moreover, it is linear with respect to $[c]$.

By a generalization of the Connes-Moscovici higher index theorem [CM to the twisted case of elliptic operators on a covering space that are invariant under the projective action of the fundamental group defined by $\sigma$, (see $[\mathrm{Ma}$ ] for a detailed proof), one has

$$
\left[\tau_{c}\right]\left(\operatorname{ind}_{\Gamma_{g}}\left(\widetilde{\not_{\mathcal{E}}^{+}} \otimes \nabla\right)\right)=\frac{1}{2 \pi}\left\langle\hat{A}\left(\Sigma_{g}\right) \operatorname{ch}(\mathcal{E}) e^{[\omega]} \psi^{*}(c),\left[\Sigma_{g}\right]\right\rangle,
$$

where $\psi: \Sigma_{g} \rightarrow \Sigma_{g}$ is the classifying map of the universal cover (which in this case is the identity map) and $[c]$ is considered as a degree 2 cohomology class on $\Sigma_{g}$. We next simplify the right hand side of $(*)$ using the fact that $\hat{A}\left(\Sigma_{g}\right)=1$ and that

$$
\begin{aligned}
\operatorname{ch}(\mathcal{E}) & =\operatorname{rank} \mathcal{E}+c_{1}(\mathcal{E}), \\
\psi^{*}(c) & =c, \\
e^{[\omega]} & =1+[\omega] .
\end{aligned}
$$

We obtain

$$
\left[\tau_{c}\right]\left(\operatorname{ind}_{\Gamma_{g}}\left(\widetilde{\not_{\mathcal{E}}^{+}} \otimes \nabla\right)\right)=\frac{\operatorname{rank} \mathcal{E}}{2 \pi}\left\langle[c],\left[\Sigma_{g}\right]\right\rangle
$$

Corollary 11. Let $c,[c] \in H^{2}\left(\Gamma_{g}\right)$, be the area cocycle. Then one has

$$
\left\langle[c],\left[\partial_{\mathcal{E}}^{+}\right]\right\rangle=2(g-1) \operatorname{rank} \mathcal{E} \in \mathbb{Z} .
$$

Proof. When $c,[c] \in H^{2}\left(\Gamma_{g}\right)$, is the area 2-cocycle, one has

$$
\left\langle[c],\left[\Sigma_{g}\right]\right\rangle=-2 \pi \chi\left(\Sigma_{g}\right)=4 \pi(g-1) .
$$

Remarks 14. These theorems have been generalised in Ma]. They agree with Xia's result [Xia], although our methods are different.

We next describe the canonical pairing of $K_{0}\left(C_{r}^{*}\left(\Gamma_{g}, \sigma\right)\right)$, given $[c] \in H^{2}\left(\Gamma_{g}\right)$. Since $\Sigma_{g}$ is negatively curved, we know from [Ji] that

$$
A_{\sigma, g}=\left\{f:\left.\Gamma_{g} \rightarrow \mathbb{C}\left|\sum_{\gamma \in \Gamma_{g}}\right| f(\gamma)\right|^{2}(1+l(\gamma))^{k}<\infty \text { for all } k \geq 0\right\}
$$

where $l: \Gamma_{g} \rightarrow \mathbb{R}^{+}$denotes the length function, is a dense and spectral invariant subalgebra of $C_{r}^{*}\left(\Gamma_{g}, \sigma\right)$. In particular it is closed under the smooth functional calculus, and is known 
as the algebra of rapidly decreasing $L^{2}$ functions on $\Gamma_{g}$. By a theorem of [Bost], the inclusion map $A_{\sigma, g} \subset C_{r}^{*}\left(\Gamma_{g}, \sigma\right)$ induces an isomorphism

$$
K_{j}\left(A_{\sigma, g}\right) \cong K_{j}\left(C_{r}^{*}\left(\Gamma_{g}, \sigma\right)\right), \quad j=0,1 .
$$

As $\Sigma_{g}$ is a negatively curved manifold, we know (by [Mos] and [Gr]) that degree 2 cohomology classes in $H^{2}\left(\Gamma_{g}\right)$ have bounded representatives i.e. bounded 2-cocycles on $\Gamma_{g}$. Let $c$ be a bounded 2-cocycle on $\Gamma_{g}$. Then it defines a cyclic 2-cocycle $\tau_{c}$ on the twisted group algebra $\mathbb{C}\left(\Gamma_{g}, \sigma\right)$, by a slight modification of the standard formula $\mathrm{CM}$, (Ma for the general case)

$$
\tau_{c}\left(f^{0}, f^{1}, f^{2}\right)=\sum_{g_{0} g_{1} g_{2}=1} f^{0}\left(g_{0}\right) f^{1}\left(g_{1}\right) f^{2}\left(g_{2}\right) c\left(1, g_{1}, g_{1} g_{2}\right) \sigma\left(g_{1}, g_{2}\right) .
$$

Here $c$ is assumed to be skew-symmetrized. Since the only difference with the expression obtained in CM is $\sigma\left(g_{1}, g_{2}\right)$, and since $\left|\sigma\left(g_{1}, g_{2}\right)\right|=1$, we can use Lemma 6.4, part (ii) in [CM and the assumption that $c$ is bounded, to obtain the necessary estimates which show that in fact $\tau_{c}$ extends continuously to the bigger algebra $A_{\sigma, g}$. This induces an additive map in $K$-theory as before:

$$
\begin{gathered}
{\left[\tau_{c}\right]: K_{0}\left(A_{\sigma, g}\right) \rightarrow \mathbb{R}} \\
{\left[\tau_{c}\right]([e]-[f])=\widetilde{\tau}_{c}(e, \cdots, e)-\widetilde{\tau}_{c}(f, \cdots, f),}
\end{gathered}
$$

where $e, f$ are idempotent matrices with entries in $\left(A_{\sigma, g}\right) \sim$ (the unital algebra associated to $\left.A_{\sigma, g}\right)$ and $\widetilde{\tau}_{c}$ is the canonical extension of $\tau_{c}$ to $\left(A_{\sigma, g}\right)^{\sim}$. Observe that the twisted Kasparov map is merely

$$
\mu_{\sigma}\left(\left[\not_{\mathcal{E}^{+}}\right]\right)=j_{*}\left(\operatorname{ind}_{\Gamma_{g}}\left(\widetilde{\partial_{\mathcal{E}}^{+}} \otimes \nabla\right)\right) \in K_{0}\left(C^{*}\left(\Gamma_{g}, \sigma\right)\right) .
$$

Here $j: \mathbb{C}\left(\Gamma_{g}, \sigma\right) \otimes \mathcal{R} \rightarrow C^{*}\left(\Gamma_{g}, \sigma\right) \otimes \mathcal{K}$ is the natural inclusion map, and $j_{*}: K_{0}\left(\mathbb{C}\left(\Gamma_{g}, \sigma\right) \otimes\right.$ $\mathcal{R}) \rightarrow K_{0}\left(C^{*}\left(\Gamma_{g}, \sigma\right)\right)$ is the induced map in $K$-theory. Therefore one has the equality

$$
\left\langle[c], \mu_{\sigma}^{-1}[P]\right\rangle=\left\langle\left[\tau_{c}\right],[P]\right\rangle
$$

for any $[P] \in K_{0}\left(A_{\sigma, g}\right) \cong K_{0}\left(C_{r}^{*}\left(\Gamma_{g}, \sigma\right)\right)$. Using the previous corollary, one has

Corollary 12. Let $c,[c] \in H^{2}\left(\Gamma_{g}\right)$, be the area 2-cocycle. Then $c$ is known to be a bounded 2-cocycle, and one has

$$
\left\langle\left[\tau_{c}\right],[P]\right\rangle=2(g-1)\left(\operatorname{rank} \mathcal{E}^{0}-\operatorname{rank} \mathcal{E}^{1}\right) \in \mathbb{Z},
$$

where $[P] \in K_{0}\left(A_{\sigma, g}\right) \cong K_{0}\left(C_{r}^{*}\left(\Gamma_{g}, \sigma\right)\right)$, and where

$$
\mu_{\sigma}^{-1}[P]=\left[\not_{\mathcal{E}^{0}}^{+}\right]-\left[\not_{\mathcal{E}^{1}}^{+}\right] \in K_{0}\left(\Sigma_{g}\right) .
$$

Remarks 15. This generalizes the main result of Xia, Xia].

We will next prove the existence of a canonical element in $K K\left(C_{r}^{*}\left(\Gamma_{g}, \sigma\right), \mathbb{C}\right)$, which we call the twisted Mishchenko element.

Theorem 16 (The twisted Mishchenko element). There exists a unique element $\left[m_{\sigma}\right] \in$ $K K\left(C_{r}^{*}\left(\Gamma_{g}, \sigma\right), \mathbb{C}\right)$, called the twisted Mishchenko element, such that

$$
[1] \otimes_{C_{r}^{*}\left(\Gamma_{g}, \sigma\right)}\left[m_{\sigma}\right]=2(g-1),
$$

where $[1] \in K_{0}\left(C_{r}^{*}\left(\Gamma_{g}, \sigma\right)\right)$ denotes the module generated by $C_{r}^{*}\left(\Gamma_{g}, \sigma\right)$. 
Proof. By the well definedness of the Kasparov intersection product [Kas2], the equation $(*)$ above defines the element $\left[m_{\sigma}\right]$ uniquely. In the next section we construct a 2 -summable Fredholm module $(F, \mathcal{H})$, which defines an element $[(F, \mathcal{H})] \in K K\left(C_{r}^{*}\left(\Gamma_{g}, \sigma\right), \mathbb{C}\right)$, and whose Chern character is the cyclic area 2-cocycle $\left[\tau_{c}\right]$, (cf. [Co2]) defined by the area 2 -cocycle $c$ on the discrete group $\Gamma$. We compute that

$$
[1] \otimes_{C_{r}^{*}\left(\Gamma_{g}, \sigma\right)}[(F, \mathcal{H})]=\operatorname{index}(F)=\tau_{c}(1,1,1)=2(g-1) .
$$

By uniqueness (proved above), we see that $\left[m_{\sigma}\right]=[(F, \mathcal{H})]$, which establishes existence.

This completes the proof of Theorem 4 and Corollary 6 because we regard index $(P F P)$ as the result of pairing an element of the K-homology of $\Sigma_{g}$ (defined by the twisted Mishchenko element) with an element of $K_{0}\left(\mathcal{B}^{\Gamma}\right) \cong K_{0}\left(C^{*}\left(\Gamma_{g}, \sigma\right)\right)$. This enables us to demonstrate the relationship between Corollary 12 and the discrete model of the hyperbolic Hall effect.

\section{A discrete Fredholm module And the Analytic index}

We have observed following Sunada that $H_{\tau}$ is an operator in the twisted algebraic group algebra $\mathbb{C}(\Gamma, \sigma)$, which is a subalgebra of $A_{\sigma, g}$. We remark that a spectral projection into a gap in the spectrum of $H_{\tau}$ is given by the smooth functional calculus applied to $H_{\tau}$. It follows from [Ji] that such spectral projections lie in $A_{\sigma, g}$. Connes constructs a Fredholm module for $\mathbb{C} \Gamma$ which can be adapted to the case of $\mathbb{C}(\Gamma, \sigma)$. In his construction the Hilbert space is the $\ell^{2}$ sections of the restriction of the spinor bundle to the orbit $\Gamma$. $u$. This space is isomorphic to $\mathcal{H}=\ell^{2}(\Gamma) \oplus \ell^{2}(\Gamma)$ under the map $\iota \oplus \iota$. The grading is the obvious one given by the $2 \times 2$ matrix $\varepsilon$. We may define the operator $F$ as in Section 7 to be multiplication by the matrix function

$$
\left(\begin{array}{cc}
0 & \varphi^{*} \\
\varphi & 0
\end{array}\right)
$$

where we restrict $\varphi$ to the orbit $\Gamma . u$.

Connes [C2 shows that the module of the previous paragraph is 2-summable for $\mathbb{C} \Gamma$. We show below using the same argument as in [Co2 that if $\lambda$ denotes the left regular $\sigma$ representation of $C^{*}(\Gamma, \sigma)$ then $[F, \lambda(\gamma)]$ is Hilbert-Schmidt. So $(\mathcal{H}, F)$ is also a 2summable module for $\mathbb{C}(\Gamma, \sigma)$. We may also exploit $\mathrm{Co} 2$ to determine explicitly the character of this Fredholm module for our case. We now summarise some of the pertinent details.

First, we are using the usual trace tr on the bounded operators on $\mathcal{H}$. Second, our module is the $\ell^{2}$ sections of the restriction of the spinor bundle to the orbit. ¿From this point of view $F$ corresponds to Clifford multiplication of a unit tangent vector to a geodesic connecting a given vertex of the graph to a point $x_{0} \notin \Gamma$.u. We use the same notation $\varphi(\gamma . u)$ for this unit tangent vector, regarding $\varphi$ as a function from $\Gamma$.u to $T(\mathbb{H})$, the tangent space of $\mathbb{H}$, as no confusion will arise. 
Next, note that for $f \in \mathcal{H}$,

$$
[F, \lambda(\gamma)] f\left(\gamma^{\prime}\right)=\left(\varphi(\gamma \cdot u)^{\prime}-\varphi\left(\gamma^{-1} \gamma^{\prime} \cdot u\right)\right) \lambda(\gamma) f\left(\gamma^{\prime}\right) .
$$

Connes observes that the operator on the RHS is Hilbert-Schmidt as a result of the convergence of the Poincare series:

$$
\sum_{\gamma \in \Gamma} \exp \left(-2 d\left(\gamma \cdot u, x_{0}\right)\right)
$$

Thus if $\gamma_{0}, \gamma_{1}, \gamma_{2}$ lie in $\Gamma$ then

$$
\operatorname{tr}\left(\varepsilon \lambda\left(\gamma_{0}\right)\left[F, \lambda\left(\gamma_{1}\right)\right]\left[F, \lambda\left(\gamma_{2}\right)\right]\right)=\frac{1}{2} \operatorname{tr}\left(\varepsilon\left[F, \lambda\left(\gamma_{0}\right)\left[F, \lambda\left(\gamma_{1}\right)\right]\left[F, \lambda\left(\gamma_{2}\right)\right]\right) .\right.
$$

Now $\lambda\left(\gamma_{0}\right)\left[F, \lambda\left(\gamma_{1}\right)\right]\left[F, \lambda\left(\gamma_{2}\right)\right]$ is the operator

$$
\left(\lambda\left(\gamma_{0}\right)\left[F, \lambda\left(\gamma_{1}\right)\right]\left[F, \lambda\left(\gamma_{2}\right)\right] f\right)(\gamma)=\zeta(\gamma) \sigma\left(\gamma_{1}, \gamma_{2}\right) \sigma\left(\gamma_{0}, \gamma_{1} \gamma_{2}\right) f\left(\left(\gamma_{0} \gamma_{1} \gamma_{2}\right)^{-1} \gamma\right) \text {, }
$$

where $\zeta(\gamma)$ denotes Clifford multiplication by

$$
\left(\varphi\left(\kappa_{0}^{-1} \gamma\right)-\varphi\left(\kappa_{1}^{-1} \gamma\right)\right)\left(\varphi\left(\kappa_{1}^{-1} \gamma\right)-\varphi\left(\kappa_{2}^{-1} \gamma\right)\right)
$$

with $\kappa_{j}=\gamma_{0} \ldots \gamma_{j}$. Finally we can now obtain a formula for the cyclic cocycle. Following the calculation on page 344 of [Co2 we find that for $\gamma_{0} \gamma_{1} \gamma_{2} \neq 1$ the character of the cocycle associated to our Fredholm module is zero while for $\gamma_{0} \gamma_{1} \gamma_{2}=1$ it is given by

$$
\operatorname{tr}\left(\varepsilon \lambda\left(\gamma_{0}\right)\left[F, \lambda\left(\gamma_{1}\right)\right]\left[F, \lambda\left(\gamma_{2}\right)\right]\right)=2 \sum_{\gamma \in \Gamma} \operatorname{trace}(\epsilon \zeta(\gamma)) \sigma\left(\gamma_{1}, \gamma_{2}\right),
$$

where 'trace' denotes the matrix trace on the Clifford algebra and we are utilising the fact that, for our choice of $\sigma$,

$$
\sigma\left(\gamma_{0}, \gamma_{1} \gamma_{2}\right)=\sigma\left(\gamma_{0}, \gamma_{0}^{-1}\right)=1
$$

Connes proves that trace $(\varepsilon \zeta(\gamma))$ is the Euclidean area of the triangle in the complex plane with vertices corresponding to the tangent vectors $\varphi\left(\kappa_{j}^{-1} \gamma\right)$. Then the additive group cocycle on $\Gamma$ given by

$$
c\left(1, \gamma_{1}, \gamma_{1} \gamma_{2}\right)=\sum_{\gamma \in \Gamma} \operatorname{trace}(\varepsilon \zeta(\gamma))
$$

is what Connes calls the 'volume' or area cocycle on $\Gamma$. Thus we find that we have computed the character of our Fredholm module to be:

$$
\tau_{c}\left(\gamma_{0}, \gamma_{1}, \gamma_{2}\right)=c\left(1, \gamma_{1}, \gamma_{1} \gamma_{2}\right) \sigma\left(\gamma_{1}, \gamma_{2}\right)
$$

for $\gamma_{0} \gamma_{1} \gamma_{2}=1$, with $\tau_{c}$ being zero when $\gamma_{0} \gamma_{1} \gamma_{2} \neq 1$ (the normalisation differs from Co2 page 295, but conforms with [CM]). This formula extends to give a non-trivial element of the cyclic cohomology of the smooth subalgebra $A_{\sigma, g}$ via the formula

$$
\tau_{c}\left(f^{0}, f^{1}, f^{2}\right)=\sum_{\gamma_{0} \gamma_{1} \gamma_{2}=1} f^{0}\left(\gamma_{0}\right) f^{1}\left(\gamma_{1}\right) f^{2}\left(\gamma_{2}\right) c\left(1, \gamma_{1}, \gamma_{1} \gamma_{2}\right) \sigma\left(\gamma_{1}, \gamma_{2}\right),
$$

for $f^{0}, f^{1}, f^{2} \in A_{\sigma, g}$.

Summarizing the discussion above, we have the first result of this section. 
Proposition 9. There is a 2-summable Fredholm module $(F, \mathcal{H})$ over $A_{\sigma, g}$ whose Chern character is given by the area cyclic 2-cocycle $\tau_{c}$. Therefore, by the index pairing in [Co2], one has

$$
\operatorname{index}(P(F \otimes I) P)=\left\langle\left[\tau_{c}\right],[P]\right\rangle
$$

where $P$ denotes a projection in $A_{\sigma, g} \otimes \mathcal{K}\left(\mathcal{H}_{1}\right)$ and $\operatorname{ind} \operatorname{dex}(P(F \otimes I) P)$ denotes the index of the Fredholm operator $P(F \otimes I) P$.

Assembling this proposition with our results from Section 10 we have:

Theorem 17. Let $P$ denote a projection in $A_{\sigma, g} \otimes \mathcal{K}\left(\mathcal{H}_{1}\right)$ Then in the notation of Corollary 12 of the previous section, one has

$$
\text { index }(P(F \otimes I) P)=2(g-1)\left(\operatorname{rank} \mathcal{E}^{0}-\operatorname{rank} \mathcal{E}^{1}\right) \in \mathbb{Z},
$$

where index $(P(F \otimes I) P)$ denotes the index of the Fredholm operator $P(F \otimes I) P$ acting on the Hilbert space $P\left(\mathcal{H} \otimes \mathcal{H}_{1}\right)$ and $\mu_{\sigma}^{-1}[P]=\left[\not_{\mathcal{E}^{0}}^{+}\right]-\left[\not_{\mathcal{E}^{1}}^{+}\right] \in K_{0}\left(\Sigma_{g}\right)$.

Corollary 13. Let $P$ be a projection into a gap in the spectrum of the discrete Hamiltonian $H_{\tau}$. Then $P \in A_{\sigma, g}$, and may be regarded as a twisted convolution operator by a function $p$ on $\Gamma$. Then in the notation of Corollary 12:

$$
\begin{aligned}
\operatorname{index}(P F P) & =\sum_{\gamma_{0} \gamma_{1} \gamma_{2}=1} p\left(\gamma_{0}\right) p\left(\gamma_{1}\right) p\left(\gamma_{2}\right) c\left(1, \gamma_{1}, \gamma_{1} \gamma_{2}\right) \sigma\left(\gamma_{1}, \gamma_{2}\right) \\
& =2(g-1)\left(\operatorname{rank} \mathcal{E}^{0}-\operatorname{rank} \mathcal{E}^{1}\right) \in \mathbb{Z}
\end{aligned}
$$

Note that this explains the integrality of the cyclic 2-cocycle,

$$
\sum_{\gamma_{0} \gamma_{1} \gamma_{2}=1} p\left(\gamma_{0}\right) p\left(\gamma_{1}\right) p\left(\gamma_{2}\right) c\left(1, \gamma_{1}, \gamma_{1} \gamma_{2}\right) \sigma\left(\gamma_{1}, \gamma_{2}\right)
$$

in two different ways: firstly as the index of the Fredholm operator $P F P$, and secondly as the topological index $2(g-1)\left(\operatorname{rank} \mathcal{E}^{0}-\operatorname{rank} \mathcal{E}^{1}\right)$, which is also clearly an integer.

\section{The NON-COMmUtative Unit DisC}

In [Klim+Les1,2] Klimek and Lesznewski have introduced a non-commutative unit disc and higher genus Riemann surfaces. Their disc algebra can be realised as a Toeplitz algebra obtained by compressing the commutative algebra of functions on the disc using the projection onto a holomorphic subspace of one of its representation spaces. We shall describe their construction in a slightly more general setting. The algebra $C_{c}(G / K)$ acts by multiplication $(f \mapsto M(f))$ on $L^{2}(G / K, \mu)$ for any quasi-invariant measure $\mu$. The group $G$ also has an induced $\sigma$ representation $W$ on this space, and we shall suppose that there is an irreducible subrepresentation on a subspace which is projected out by $P$. (This is certainly true in the case considered in [Klim+Les1].) The algebra $P M\left(C_{c}(G / K)\right) P$ then gives the non-commutative analogue of $C_{c}(G / K)$. Now, by definition $G$ also acts and 
therefore defines automorphisms of this algebra. Since it commutes with $P$ the covariance algebra $P M\left(C_{c}(G / K)\right) P \rtimes G$ is the same as $P\left(C_{c}(G / K) \rtimes G\right) P$, which is the compression of the imprimitivity algebra $\mathcal{A}=C_{c}(G / K) \rtimes G$. For higher genus surfaces one simply takes the $\Gamma$-invariant part of $P C_{c}(G / K) P$, which is consistent with our constructions above.

Suppose now that the irreducible subspace is defined by a reproducing kernel. Invariance of the kernel means that it is defined by twisted convolution with a continuous $\sigma$-positive definite function $\xi_{P}$ or, equivalently, that

$$
P=W\left(\xi_{P}\right)=\int \xi_{P}(g) W(g) d g .
$$

Now observe that $\xi_{P}$ can be identified with an element of the imprimitivity algebra so the covariance algebra can be identified with the compression $\xi_{P} * \mathcal{A} * \xi_{P}$ of the imprimitivity algebra.

In the cases of interest $\xi_{P}$ is the $\sigma$-positive-definite function associated with a $C^{\infty}$ vector, and so is smooth. This means that the natural module $\xi_{P} * \mathcal{M}$ for $\xi_{P} * \mathcal{A} * \xi_{P}$ retains the structure of a Fredholm module.

\section{REFERENCES}

[At] M.F. Atiyah, Elliptic operators, discrete groups and Von Neumann algebras, Astérisque no. 32-33 (1976), 43-72.

$[\mathrm{Av}+\mathrm{K}+\mathrm{P}+\mathrm{S}] \quad$ J. Avron, M. Klein, A. Pnueli, L. Sedun, Hall conductance and adiabatic charge transport of leaky tori, Phys. Rev. Lett. 69 (1990), 128-131.

$[\mathrm{Av}+\mathrm{S}+\mathrm{S}] \quad$ J. Avron, R. Seiler, B. Simon, Charge deficiency, sharge transport and comparisons of dimension, Commun. math. Phys. 159 (1994), 399-422.

$[\mathrm{Av}+\mathrm{S}+\mathrm{Y}] \quad$ J. Avron, R. Seiler, I. Yaffe, Adiabatic theorems and applications to the integer quantum Hall effect, Commun. math. Phys. 110 (1987), 33-49.

[Bel] J. Bellissard, K-theory of $C^{*}$-algebras in solid state physics, Springer Lecture Notes in Physics 257 (1986), 99-156.

$[\mathrm{Bel}+\mathrm{E}+\mathrm{S}] \quad$ J. Bellissard, A. van Elst, H. Schulz-Baldes, The non-commutative geometry of the quantum Hall effect, J. Math. Phys. 35 (1994), 5373-5451.

[Bost] J. Bost, Principe d'Oka, $K$-théorie et systémes dynamiques non commutatifs, Invent. Math. 101 (1990), no. 2, 261-333.

[BrSu] J. Brüning, T. Sunada, On the spectrum of gauge-periodic elliptic operators. Méthodes semi-classiques, Vol. 2 (Nantes, 1991). Astérisque 210 (1992), 65-74.

[CGT] J. Cheeger, M. Gromov, M. Taylor, Finite propogation speed, kernel estimates for functions of the Laplace operator and the Geometry of complete Riemannian manifolds, Jour. Diff. Geom. 17 (1982), 15-54.

[CEY] M. Choi, G. Elliott, N. Yui, Gauss polynomials and the rotation algebra, Invent. Math. 99 (1990), no. 2, 225-246.

[Comtet] A. Comtet, On the Landau levels on the hyperbolic plane, Ann.Phys. 173 (1987), 185209.

$[$ Comtet $+\mathrm{H}] \quad$ A. Comtet, P. Houston, Effective action on the hyperbolic plane in a constant external field, J. Math. Phys. 26 (1985), 185-191.

[Co] A. Connes, Non commutative differential geometry, Publ. Math. I.H.E.S. 62 (1986), 257360.

[Co2] A. Connes, Noncommutative geometry. Academic Press, Inc., San Diego, CA, (1994).

[CM] A. Connes, H. Moscovici, Cyclic cohomology, the Novikov conjecture and hyperbolic groups, Topology 29 (1990), 345-388. 
$[\mathrm{Cu}]$

[Elliott]

[Green]

[GH]

[Gr]

[Gr2]

[Helg]

$[$ Iengo $+\mathrm{Li}]$

[Ji]

[JuKas]

[Kas1]

[Kas2]

[Kas3]

[Klim+Les1]

[Klim+Les2]

[Ma]

[MC]

[Mos]

$[\mathrm{M}+\mathrm{R}+\mathrm{W}]$

$[\mathrm{Nak}+\mathrm{Bel}]$

[PR]

[PR1]

[PR2]

[Ren1]

[Ren2]

[Ren3]

J. Cuntz, K-theoretic amenability for discrete groups, J. Reine Angew. Math. 344 (1983), 180-195.

G. Elliott, On the $K$-theory of the $C^{*}$-algebra generated by a projective representation of a torsion-free discrete group, Operator Algebras and Group Representations, Pitman, London, (1983), 157-184.

P. Green, The structure of imprimitivity algebras, J. Func. Anal. 36 (1980), 88-104.

P. Griffiths and J. Harris, Principles of algebraic geometry, Wiley, New York, 1978.

M. Gromov, Volume and bounded cohomology, Publ. Math. I.H.E.S. 56 (1982), 5-99.

M. Gromov, Kähler-hyperbolicity and $L^{2}$ Hodge theory, J. Diff. Geom. 33 (1991), 263292.

S. Helgason, Differential Geometry, Lie Groups and Symmetric Spaces, Pure and Applied Mathematics, 80 Academic Press, Inc. [Harcourt Brace Jovanovich, Publishers], New York-London, (1978).

R. Iengo, D. Li, Quantum mechanics and the quantum Hall effect on Riemann surfaces, Nuclear Phys. B 413 (1994), 735-753.

R. Ji, Smooth dense subalgebras of reduced group $C^{*}$-algebras, Schwartz cohomology of groups and cyclic cohomology, Jour. Func. Anal. 107 (1992), 1-33.

P. Julg, G. Kasparov, Operator $K$-theory for the group $\mathrm{SU}(n, 1)$. J. Reine Angew. Math. 463 (1995), 99-152.

G. Kasparov, Lorentz groups, $K$-theory of unitary representations and crossed products, Soviet Math. Dokl. 29 (1984), 256-260.

G. Kasparov, $K$-theory, group $C^{*}$-algebras and higher signatures, Conspectus, (1980), published in 'Novikov conjectures, index theorems and rigidity', vol. 1, editors S. Ferry, A. Ranicki, J. Rosenberg, Lond. Math. Soc. Lecture Note Series 226 (1995), Cambridge University Press.

G. Kasparov, Equivariant $K K$-theory and the Novikov conjecture, Inv. Math. 91 (1988), 147-201.

S. Klimek, A. Lesznewski, Quantum Riemann surfaces I: the unit disc, Commun. Math. Phys. 146 (1992), 105-122.

Phys. 24 (1992), 125-139.

V. Mathai, in preparation.

P. McCann, A. Carey, A discrete model of the integer quantum Hall effect, Publ. RIMS, Kyoto Univ. 32 (1996), 117-156.

G. Mostow, Strong rigidity of symmetric spaces, Ann. Math. Studies, 78 (1973), Princeton University Press.

P. Muhly, J. Renault, P. Williams, Equivalence and isomorphism for groupoid $C^{*}-$ algebras, J. Operator Th. 17 (1987), 3-22.

S. Nakamura, J. Bellissard, Low energy bands do not contribute to the quantum Hall effect, Commun. Math. Phys. 131 (1990), 283-305.

J. Packer, I. Raeburn, Twisted cross products of $C^{*}$-algebras, Math. Proc. Camb. Phil. Soc. 106 (1989), 293-311.

J. Packer, I. Raeburn, On the structure of twisted group $C^{*}$-algebras, Trans. Amer. Math. Soc. 334 (1992), 685-718.

J. Packer, I. Raeburn, Twisted cross products of $C^{*}$-algebras. II, Math. Ann. 287 (1990), 595-612.

J. Renault, A groupoid approach to $C^{*}$-algebras, Lecture Notes in Mathematics 793 (1980), Springer, Berlin.

J. Renault, Représentations des produits croisés d'algèbres de groupoïdes, J.Operator Th. 18 (1987), 67-97.

J. Renault, The ideal structure of groupoid crossed product $C^{*}$-algebras, J.Operator Th. 25 (1991), 3-36. 
[Rief] M. Rieffel, $C^{*}$-algebras associated with irrational rotations, Pac. J. Math. 93 (1981), 415-429.

[Ros] J. Rosenberg, Continuous trace algebras from the bundle theoretic point of view, Jour. Aus. Math. Soc. 47 (1989), 368-381.

[Sh] M. Shubin, Discrete Magnetic Schrodinger operators, Comm. Math. Phys. 164 (1994), no.2, 259-275.

[Si] I.M. Singer, Some remarks on operator theory and index theory, Springer Lecture Notes in Math. 575 (1977), 128-137.

[Sun] T. Sunada, A discrete analogue of periodic magnetic Schrödinger operators, Contemp. Math. 173 (1994), 283-299.

[Tak] M. Takesaki, Theory of operator algebras I, Springer-Verlag, New York (1979).

[Xia] J. Xia, Geometric invariants of the quantum hall effect, Comm. Math. Phys. 119 (1988), 29-50.

Department of Mathematics, University of Adelaide, Adelaide 5005, Australia

E-mail address: acarey@maths.adelaide.edu.au

Department of Mathematics, University of Oxford, England.

E-mail address: khannabu@maths.adelaide.edu.au

Department of Mathematics, University of Adelaide, Adelaide 5005, Australia

E-mail address: vmathai@maths.adelaide.edu.au

Department of Mathematics, University of Adelaide, Adelaide 5005, Australia

E-mail address: pmccann@maths.adelaide.edu.au 